University of Redlands

\title{
Differential Leveling Conversion and Analysis Toolset
}

\author{
A Major Individual Project submitted in partial satisfaction of the requirements \\ for the degree of Master of Science in Geographic Information Systems \\ By \\ Lisa Berry \\ Ruijin Ma, Ph.D., Committee Chair \\ Douglas M. Flewelling, Ph.D.
}

December 2013 
l'he report of Lise Therty is enproverit.

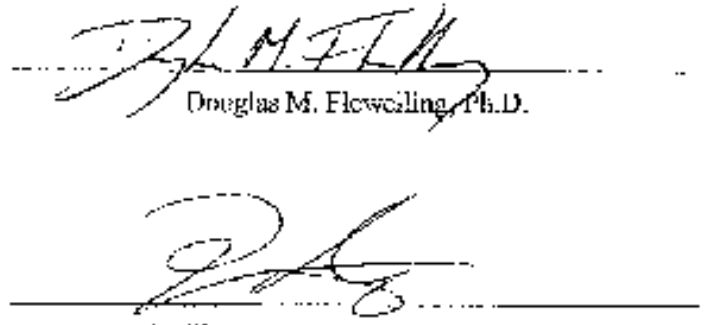

Ruịjan Ma, l'h.D.. (sımımilles Chair-

Duccrber 2013 
Differential Leveling Conversion and Analysis Toolset

Copyright (C) 2013

by

Lisa Berry 





\section{Acknowledgements}

This project would not have been a success if not for many important people encountered throughout the process. While some built me up, other tore me down. But the most important lessons I learned were not necessarily skills regarding GIS, but how to handle the ups and downs of life.

I must first thank my parents, who without their support and encouragement through the years, I would have never reached a goal such as this. They have been my foundation and they have been my wings. I would be nothing without them.

I must also thank the friends I have gained through this year and acknowledge their emotional and academic help through the process. Whether it be nights at Romano's or burgers by the pool, I have created some of my most valuable memories this year and I have gained a couple of the best friends I could ever ask for.

The faculty and staff of the MS GIS program, along with adjunct professors from Esri, provided the tools and assistance necessary for the completion of this project. Without them, I would still be completing elementary GIS buffers and geoprocessing rather than creating GIS solutions for a client.

While it may seem odd, I would like to acknowledge my enemies and my struggles. While you tried various times to break my spirit or demolish my motivation, you also taught me to be stronger than I thought possible. You taught me to dig deeper into myself and tear down walls that bound me and my potential. If not for the low points, we can't truly appreciate the successful and joyous moments of our lives. 



\author{
Abstract \\ Differential Leveling Conversion and Analysis Toolset \\ by \\ Lisa Berry
}

As Caltrans is transitioning their spatial analysis and management of surveying data into a GIS, the process of converting original files into compatible formats is tedious and time-consuming. This project develops a geodatabase and creates a tool capable of converting and processing survey observations into the geodatabase. While maintaining all the original observations, the developed geodatabase allows efficient data retrieval and analysis of changes of elevation differences among benchmarks over time. The developed tool and geodatabase will be used by Caltrans to manage their surveying data along the Southern California transportation infrastructure. 



\section{Table of Contents}

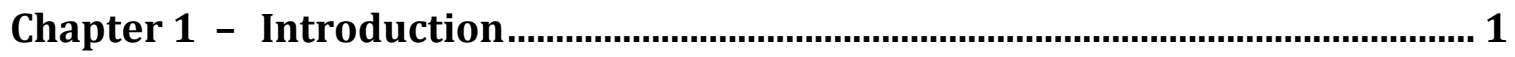

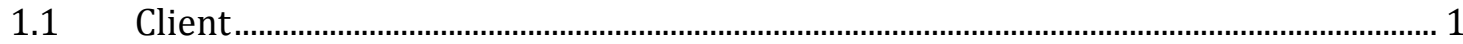

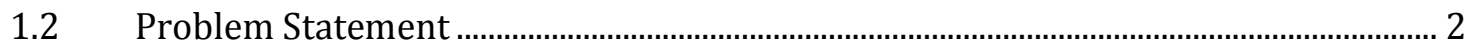

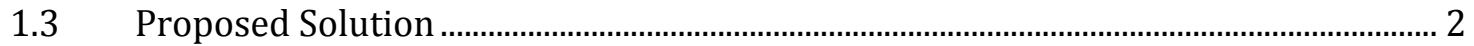

1.3.1 Goals and Objectives ............................................................................................................ 2

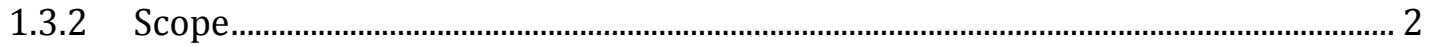

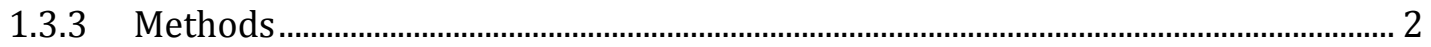

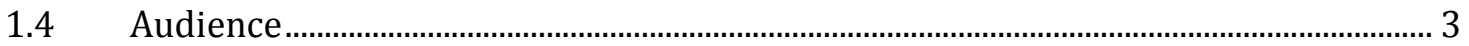

$1.5 \quad$ Overview of this Report ....................................................................................................... 3

Chapter 2 - Background and Literature Review …................................................. 5

$2.1 \quad$ Differential Leveling ................................................................................................ 5

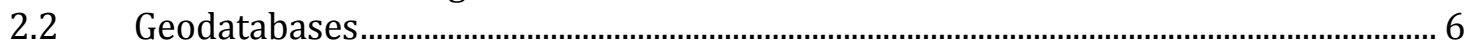

$2.3 \quad$ NGS Blue Book and Reduce Format ................................................................................. 7

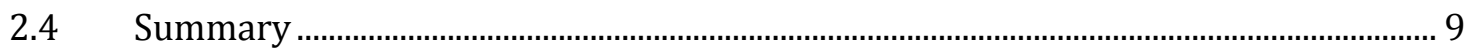

Chapter 3 - Systems Analysis and Design ……...................................................11

3.1 Problem Statement ...........................................................................................................11

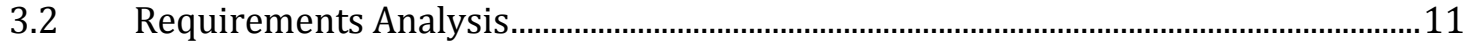

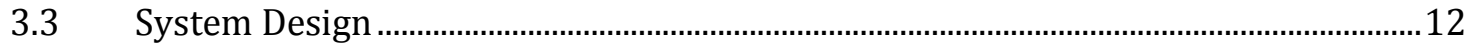

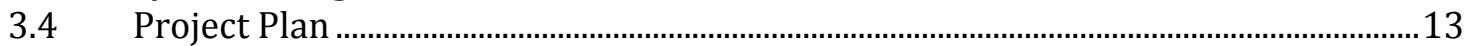

3.4.1 Requirement Analysis ...................................................................................................

3.4.2 Database Creation ...............................................................................................................14

3.4.3 Prototype Development and Implementation...............................................................15

3.4.4 Documentation ....................................................................................................................15

3.4.5 Deliverables ....................................................................................................................

3.5 Risk Analysis and Mitigation Planning …………………………………………………...16

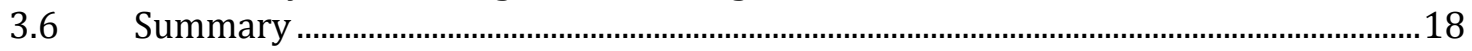

Chapter 4 - Database Design ................................................................................19

$4.1 \quad$ Conceptual Data Model......................................................................................................19

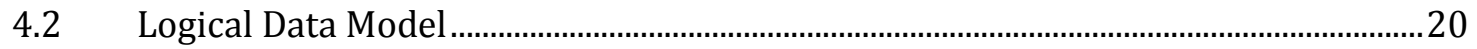

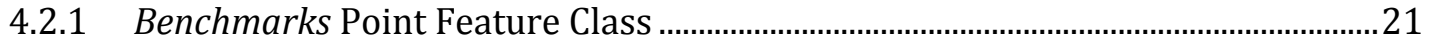

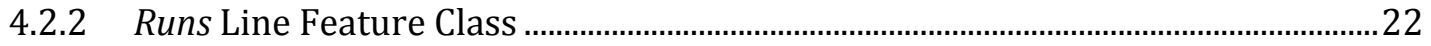

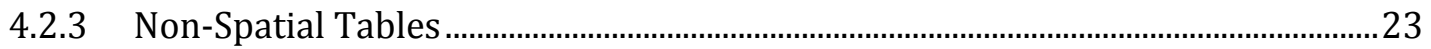

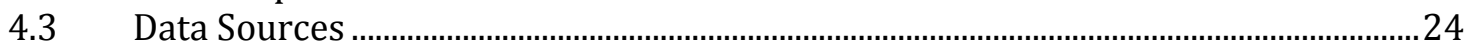

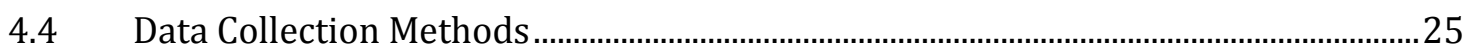

$4.5 \quad$ Data Scrubbing and Loading ...........................................................................................25

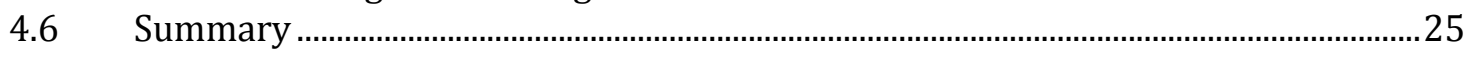

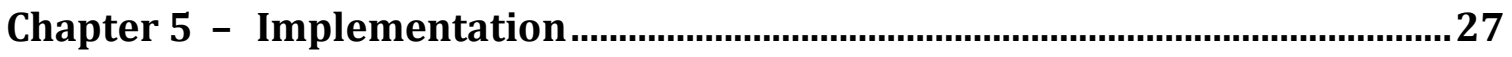

$5.1 \quad$ Geodatabase Creation ..................................................................................................2

5.2 Basic Dataset Prepping Using Python ………………………………………………....22

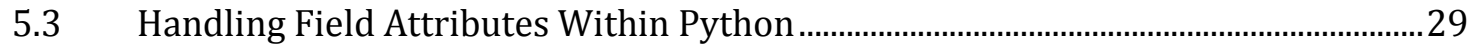

5.4 Geodatabase Loading using Python .................................................................................31

5.5 Handling Repeated Surveys and Non Blue Book Files ....................................................33

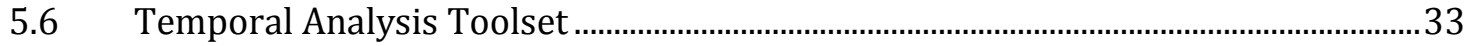

5.6.1 Recognizing Similar Line Segments ...................................................................... 


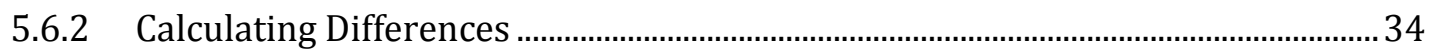

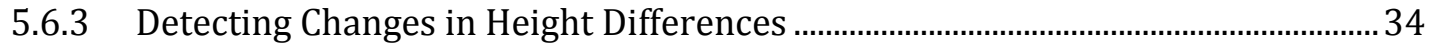

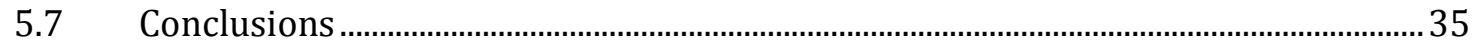

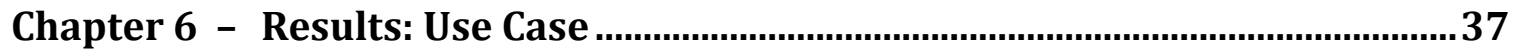

$6.1 \quad$ Graphical User Interface

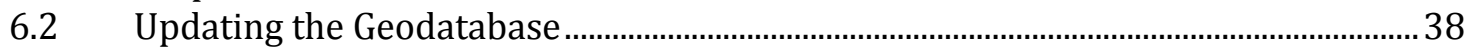

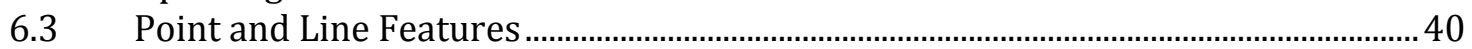

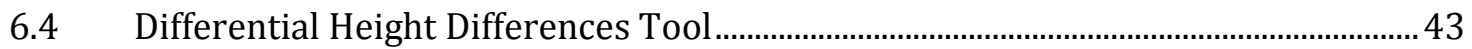

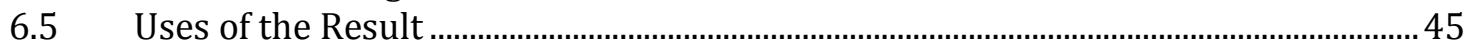

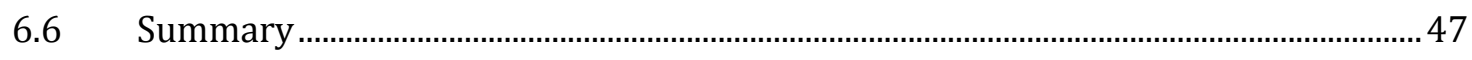

Chapter 7 - Conclusions and Future Work .........................................................49

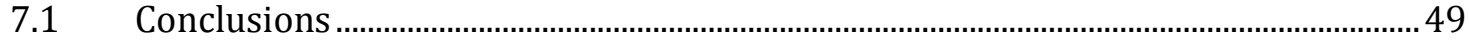

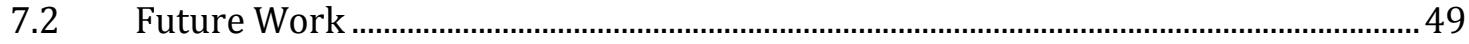

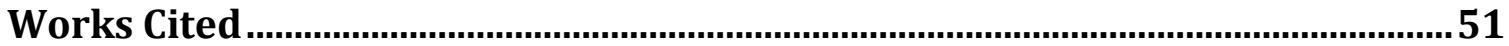

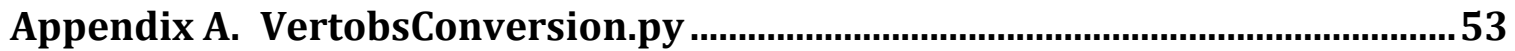

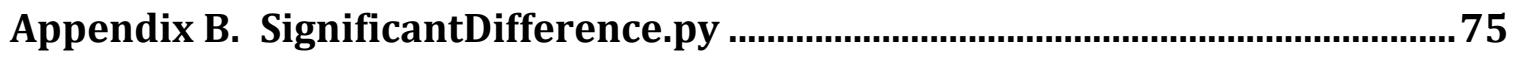

Appendix C. Reduce (Reduc6) Format Specifications.......................................... 79 


\section{Table of Figures}

Figure 1.1: Overall Methodology of Project Implementation .................................... 3

Figure 2.1: Differential Leveling Between Two Benchmarks .................................. 6

Figure 2.2: HGZ Format Conversion (National Oceanic and Atmospheric

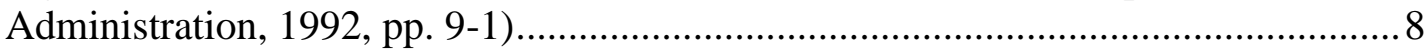

Figure 3.1: Overall System Design Process......................................................... 13

Figure 3.2: Process for Determining Requirements.............................................. 14

Figure 3.3: Geodatabase Creation Workflow ........................................................... 15

Figure 3.4: Prototype and Implementation Workflow ........................................ 15

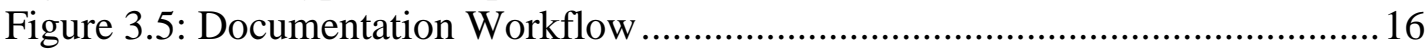

Figure 4.1: Conceptual Model for the Dataset......................................................... 19

Figure 4.2: Basic Structure of Geodatabase........................................................2 21

Figure 4.3: Example of Benchmark Field and Field Data Type ..............................22

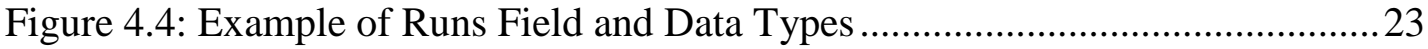

Figure 4.5: Example of Survey Field and Data Types..........................................23

Figure 4.6: Overall Relationship of Geodatabase Tables and Tools .........................24

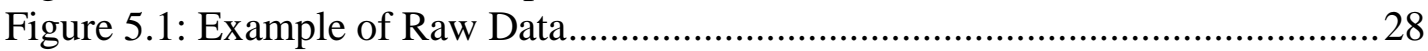

Figure 5.2: Handling Different Features Based on Line Record Type ......................28

Figure 5.3: Parsing and Stripping the Raw Data................................................29

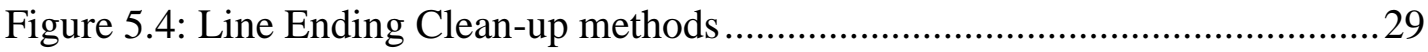

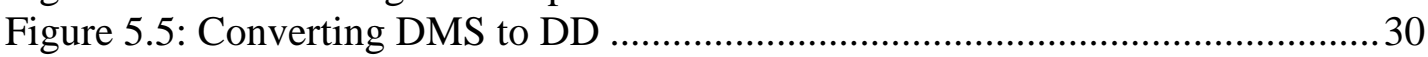

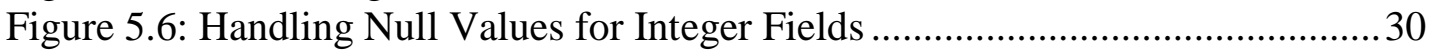

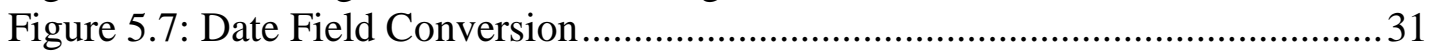

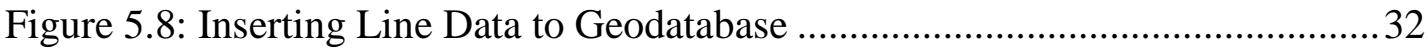

Figure 6.1: Graphical User Interface of the Conversion Toolset ...............................37

Figure 6.2: Benchmarks Table Attributes After Running Conversion Tool.............. 38

Figure 6.3: Runs Table Attributes After Running Conversion Tool ..........................39

Figure 6.4: Non-Spatial Table within Geodatabase and Key Value ......................... 40

Figure 6.5: Point and Line Features Created By Tool ............................................. 41

Figure 6.6: Select By Attributes Use Case................................................................ 42

Figure 6.7: Select By Attribute Use Case Result .................................................. 43

Figure 6.8: Significant Differences Temporal Analysis Tool..................................43

Figure 6.9: SigDifference Table Filled with Analysis Tool ....................................44

Figure 6.10: Highlighted Runs with Significant Differences over Time ...................45

Figure 6.11: Oil and Gas Basin Overlay with Height Change Results ......................46

Figure 6.12: Water Basin Overlay with Height Change Results ..............................46

Figure 6.13: Sediment Basin Overlay with Height Change Results .........................47 



\section{List of Tables}

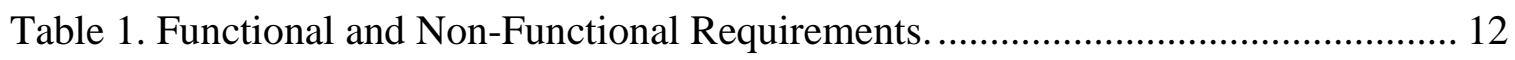

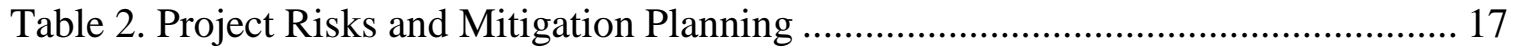





\section{List of Acronyms and Definitions}

$\begin{array}{ll}\text { Benchmarks } & \text { Stable reference points used to perform geodetic surveys } \\ \text { Blue Book } & \text { Specifications set by NGS for recording Geodetic Surveys } \\ \text { Caltrans } & \text { California Department of Transportation } \\ \text { GIS } & \text { Geographical Information Systems } \\ \text { GUI } & \text { Graphical User Interface } \\ \text { NGS } & \text { National Geodetic Survey } \\ \text { NOAA } & \text { National Oceanic and Atmospheric Administration } \\ \text { PID } & \text { Point Identification number } \\ \text { Run } & \text { A measurement/distance between two benchmarks } \\ \text { SSN } & \text { Station Serial Number } \\ \text { VERT OBS } & \text { Vertical Observation Data }\end{array}$





\section{Chapter 1 -Introduction}

The infrastructure of the southern California transportation system is managed using technologies such as geographic information systems (GIS) in order to evaluate the stability of a road system in a given area. Using datasets containing elevation changes over time allows agencies such as the California Department of Transportation (Caltrans) to compare elevations temporally along surveyed routes. This project enables Caltrans to perform spatial and temporal analysis by implementing a GIS solution for the agency's workflow. This project streamlines the process for dataset analysis in order for Caltrans to make more informed decisions regarding the transportation structure in southern California.

Caltrans spends massive amounts of time and resources analyzing changes in elevation by surveying the same region over time, but the datasets needed to be analyzed and converted to a usable format. The purpose of this project was to develop a tool to convert the survey data to GIS data format and developed a geodatabase to manage the data for analyses.

Caltrans works with surveyors to collect elevation data in the Los Angeles and Ventura areas through differential leveling. As data accumulates through time, Caltrans needs to be able to examine elevation and geological data together to assess the stability of the transportation infrastructure in southern California. Caltrans wants to discover weaknesses in the transportation infrastructure by analyzing the elevation data near main roads.

Benchmarks provide stable locations over time and differential leveling measures the elevation differences among these benchmarks. The tool developed herein enables survey datasets from the National Geodetic Survey (NGS) to be incorporated with many GIS layers for the Southern California region within a GIS. Ultimately, the product allows Caltrans to correlate the differential leveling measurements with natural geomorphological processes.

For this project, Caltrans provided a pilot dataset of such surveying observations in the NGS text format dating back to the 1960s, and required a tool to convert the observations into a geodatabase for easy management. The goal was to use the historical data to create a toolset capable of completing temporal elevation comparisons over time.

\subsection{Client}

The main point of contact for this project was Mr. Jay Satalich from the Southern California regional branch of Caltrans. The client worked directly with team members from NGS and Esri to clarify questions regarding the datasets and how they need to be used. The client provided the expertise for surveying collection methods and temporal analyses in order to clarify the requirements of this project. The client also provided a sample dataset of the format that required conversion into Esri GIS format data. Communication with the client allowed the project to be completed on time, and allowed for different ideas to be accepted or denied throughout the process. 


\subsection{Problem Statement}

The client's main problem was their limited ability to organize and maintain existing spatial data and data that will be collected in the future. The client previously performed analyses by hand, so they needed a more efficient method to convert and analyze their datasets spatially. The ability to use GIS will save the agency time and money by making analysis of survey observations more efficient. Since they used a nationwide format, this project not only solved this problem for the client, but for anyone using the same format.

\subsection{Proposed Solution}

The client required an ArcGIS tool that would convert their Blue Book formatted HGZ text files into a geodatabase. Such a geodatabase would contain all tools, feature classes, and tables necessary for the client to quickly analyze their survey data. Overall, the geodatabase unifies the workflow for conversion and analyses process for the Blue Book formatted files.

This project helped the client by creating a tool which would automatically convert their files into the desired ArcGIS compatible format. To streamline their analyses, this project also created a tool within the geodatabase to perform temporal analyses on the elevation changes of the benchmarks over time. This tool detects areas of elevation changes and allows the client to potentially recognize areas of instability over time.

\subsubsection{Goals and Objectives}

The goal of this project was to help Caltrans transition their surveying datasets into a GIS setting to allow them to perform temporal analyses of elevation change. One objective was to create a geodatabase to manage the survey data in a GIS compatible format and perform temporal analyses. The second objective was to develop a tool to convert the survey data into GIS data format. Prior to this project, the format of the survey data was difficult to use and had limited utility of spatial analyses for the client. When converted into a geodatabase, the differential leveling survey information could be visualized, analyzed, and stored more efficiently than the Blue Book format.

\subsubsection{Scope}

The final deliverable for this project was a transferable geodatabase contained within ArcGIS 10.1. The geodatabase contains the file conversion tool, the change analysis tool, the feature classes of the survey data, and tables storing survey data. The conversion tool used Python and ESRI Arcpy module to convert files in Blue Book format to feature classes and tables in the geodatabase. The change analysis tool was developed to identify and retrieve differential leveling data with temporal changes larger than a threshold specified by the user.

\subsubsection{Methods}

This project was completed solely in ArcGIS 10.1 and Python, and worked with version 10.1 geodatabase structures. The client provided nine sample HGZ files to use as a pilot 
dataset. Each file contained differential leveling data from one survey project. The HGZ files were stored in a Blue Book format, which was described in a metadata file provided by the client. The metadata file detailed different types of observations and data that could exist in a Blue Book file. It also helped create the conceptual model of the developed geodatabase. The geodatabase schema was created considering the temporal analysis the client needed to perform on the dataset. The geodatabase schema was created, a Python script was written to convert Blue Book files to feature classes and data tables into the geodatabase. An ArcGIS tool within the geodatabase was created to connect the developed Python script with ArcGIS functionalities. The survey data had to be converted into point and line features, and also had to have a quality control system to ensure the information was converted correctly.

Once the Blue Book files were successfully converted into GIS compatible format, a temporal analysis tool was developed to identify and retrieve data from benchmarks with large elevation changes over time. A threshold is provided by the user. The tool located areas with elevation changes larger than the threshold over time. An overall outline of the process is displayed in Figure 1.1.

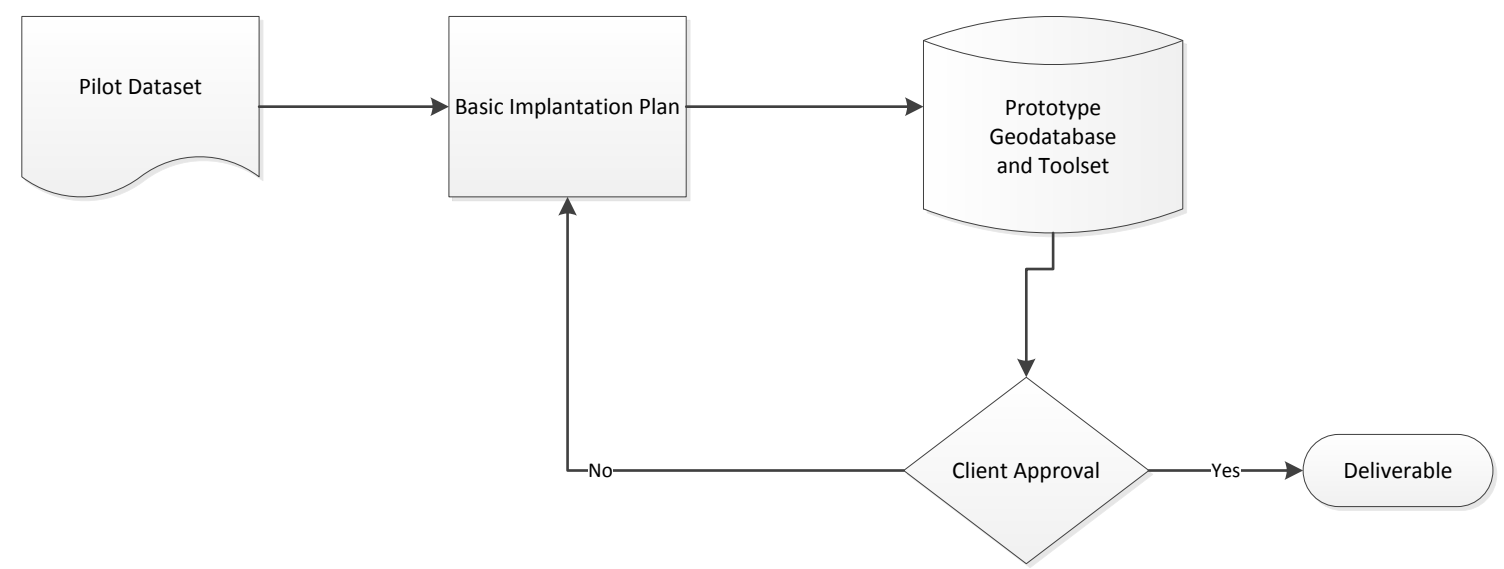

Figure 1.1: Overall Methodology of Project Implementation

\subsection{Audience}

While the initial audience for this report is the client, Caltrans, this project is applicable towards a nationwide dataset conversion process. Since the Blue Book format is a nationwide format supported by NGS, the audience of this project could be surveyors familiar with the Blue Book format, or GIS Analysts who use the toolset in order to transform their files into a GIS. This paper allows a general audience knowledgeable in GIS technologies to understand the methods of how this project was completed, and thus shows that it is a repeatable process for GIS users.

\subsection{Overview of this Report}

This report documents the background, methods, and conclusions of this project. Chapter 2 contains background and literature highlights of similar works and verifies why this project was necessary. Chapter 3 reviews the system design for this project and defines the methods and requirements. Chapter 4 provides a detailed explanation of the database 
design and data cleanup. Chapter 5 covers the implementation process for this project, which includes a detailed description of the steps taken to complete this project. Chapter 6 covers the analysis performed by the project and a use case of the results. Chapter 7 describes potential future work that could be completed based on the work of this project as well as the project's conclusions. 


\section{Chapter 2 -Background and Literature Review}

As technology has become afforable and more advanced, the techniques of surveying have been directly impacted. With advancements such as global positioning systems (GPS) and ArcGIS, surveyors have adjusted their methods in the field to match the growth of technology, but classic surveying techniques continue to be important. The main client, Caltrans, uses differential leveling for all "Caltrans-involved transportation improvement projects" (California Department of Transportation, 2006, p. 1), and are set to specific standards by the Federal Geodetic Control Subcommittee. Organizing and analyzing past differential leveling surveys allows Caltrans to focus their attention and resources on areas with major height changes over time. This helps direct funding for the transportation infrastructure to the places with the most need. While survey datasets existed in a digital format prior to this project, they were not compatible with any GIS system, such as ArcMap. This chapter provides a review of differential leveling and the importance of transitioning the datasets into a GIS.

\subsection{Differential Leveling}

The ability to track changes in the landscape makes surveying a vital tool for the transportation infrastructure in the United States. The ability to differentiate between stable and changing elevations helps focus state funds to the most suitable areas. The foundation of surveying is precise measurement, and placing a dataset into a GIS must maintain this quality in order to properly use surveying observations. By digitizing existing paper scaled maps, survey datasets can now be analyzed with computer-aided drafting (CAD) programs and other GIS systems. However, distances, locations, areas, elevations, and bearings can only be as precise as the original source of information (Estopinal, 2009, p. 132). Loading survey points into a GIS allows observations to be shared and analyzed, but they must be kept precise in order to be useful for surveying analyses.

While the majority of past and present surveying involved parcel measurement, Caltrans worked with larger scale measurements and had to consider geodetic distortions over a network. Similar network analyses were completed by Satalich (2010) within a survey request covering the benchmarks following the Southern California Interstate 5 freeway. Within this report, Satalich performed a regression analysis using "good" benchmarks to validate differential leveling points within a network from past measurements. The original format of the client's pilot dataset made pinpointing particular benchmark information possible, but difficult with hand calculations. This project simplifies the process of the client's calculations by storing the datasets within a geodatabase. The product of this project allows the client not only to perform basic calculations, but also perform spatial analyses on the locations of the surveys.

The methods of differential leveling are set by the Federal Geodetic Control Subcommittee, and specify the processes and standards that should be observed when performing a survey. Measurements are taken between benchmarks, which are described within the Caltrans Surveys Manual as being "of a stable, permanent nature" and "conveniently located and easily accessible" (California Department of Transportation, 
2006, pp. 8-2). The differential leveling measurement ultimately records a relative height difference between two benchmark locations. As explained within NGS Vertical Observation standards, a Station Serial Number (SSN) is used to identify benchmarks within a particular survey, and a Point Identification (PID) number specifies an NGS recognized benchmark location. Within these surveys, the raw observations are stored within a running record, which stores the differential height information. Figure 2.1 shows two benchmarks and the process of using survey equipment to measure the differential height between them (Transportation, 2011).

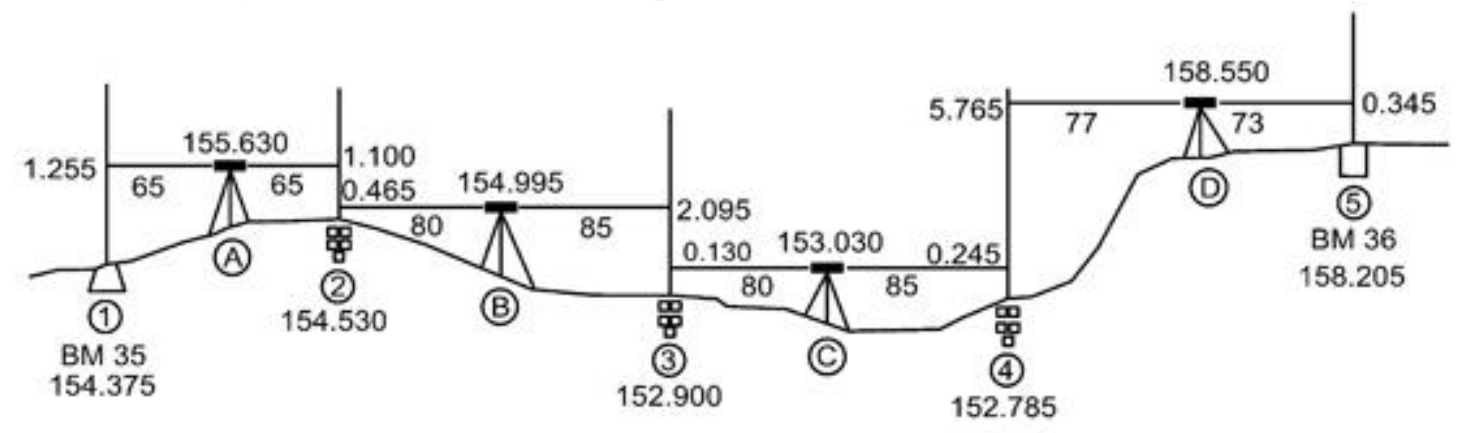

Figure 2.1: Differential Leveling Between Two Benchmarks

Raw survey observations require several corrections to analyze the values taken during a survey. These corrections consider factors such as temperature, time of day, equipment calibration, among others. Locating observation errors and anomalies is an important part of using surveyed information. In the past, errors within surveying were found by tedious calculations and regression analyses. For example, Ross Stein et al. (1986) used refraction models to prove the necessity of a temperature correction equation to eliminate refraction error caused by temperature changes while taking survey measurements. The process to perform necessary calculations is slow, inefficient, and requires a large amount of time. Placing observations within a GIS speeds up the process of analyses and allows the client to spatially perform analysis on the locations surveyed. Surveying datasets contain large amounts of information related to the raw survey observations. The factors such as temperature and raw measurements are used to evaluate the surveys, and can be more efficiently analyzed when stored within a database. Visualizing and performing spatial analysis provides the client with additional insight, and allows them to make more informed decisions.

\subsection{Geodatabases}

In the past, the ability to store and access large amounts of survey observations could be costly and time-consuming. By placing a dataset into a geodatabase, relationships within the date can be maintained and reused in future analysis.

Caltrans provided a pilot set of differential leveling observations in Blue Book format, which had to be transferred into a geodatabase. This pilot dataset covered the southern California region and dated back to the 1960s. When creating a geodatabase for transportation purposes, "a geodatabase must accommodate the many segmentation schemes employed and the various linear and coordinate referencing systems available to show where the elements, conveyances, and characteristics of transportation systems are 
located" (Butler, 2008, p. 2). The features and data within the surveying files intersect and maintain relationships with the southern California transportation infrastructure. The height differences measured between benchmarks in a survey correspond with locations along infrastructures. A proper geodatabase would allow overlaying survey observations with the infrastructure to locate possible unstable regions.

Leach (2007) investigated the process of uploading survey observations into a database, but technology has advanced since his research was completed. Leach used Python coding to create a geodatabase specific to his client's needs and also gave them the "capability to make local customizations or database design changes" (Leach, 2007, p. 14). Editing a geodatabase using Python scripts allows for customized and specific processes to occur when loading tables or feature classes. In 2009, Torres-Ruiz created a geodatabase conversion tool using ModelBuilder within ArcGIS. He used ArcGIS 9.3 where many of the methods for the creation of his application remain the same as ArcGIS 10.1 , which is the license held by the client and the basis of this project. Torres-Ruiz created a database schema using Unified Markup Language (UML) in Microsoft Visio 2007 and ArcGIS Diagrammer. While there are major differences in the data types between the two projects, Torres-Ruiz simplifed his geodatabase management by using ArcGIS ModelBuilder to load his spreadsheet and, "append the data into the desired feature class" (Torres-Ruiz, 2009, p. 4). There is more than one method to place files into a geodatabase, but the method should ultimately be determined by the data source. In their projects, organized spreadsheets of data allowed for out-of-the box ArcGIS conversion. For this project, the pilot dataset provided by the client was in a text format, which required a large amount of data scrubbing and handling.

\subsection{NGS Blue Book and Reduce Format}

As technology has changed, GPS has become the predominant form of data collection for surveying datasets. The NGS Input Formats and Specification of the National Geodetic Survey Data Base ("Blue Book") contains the guidelines for all standards necessary to record geodetic surveys. Differential leveling uses Vertical Observation (VERT OBS) data collection methods, which hold specific guidelines set by the NGS Blue Book standards. The differential leveling records held by the client are all VERT OBS records and followed the NGS Blue Book format. According to this document, there are four categories in which VERT OBS data are organized: Line Identification Data, Survey Equipment Data, Field Abstract Data, and Observation Data. As explained within the document, "the respective data have been grouped into one or more logical units called "records" "which contain 80 characters or "card columns" (National Oceanic and Atmospheric Administration, 2004). This document records the basic positioning and organization of the records within each VERT OBS dataset. This Blue Book provides a scehma for deciphering the datasets provided by the client.

An additional document for specifying the dataset details is the Reduce Record Format given by NGS. This document contains more details regarding the metadata and positioning for each line within records. Similar to the NOAA document, this document specifies the various required and optional fields within each line of the HGZ files storing the information. The Reduce Record Format document describes the basis for each unique survey, stating that each line accepts an, "accession (job) numbers of 13 characters max" as a combination of "alpha-numeric" characters (National Geodetic 
Survey, 2001). The Reduce document provides a key for parsing the line records and was used to determine line indexing specifications that were used within the Python script. The script used these indexes in order to read and separate attributes within the HGZ files.

The NGS Blue Book and Reduce format document provided detailed descriptions for line records. These documents are the keys used for determining attribute data within the line records and are described in later chapters.

As described throughout this document, the pilot dataset was comprised of a text file formatted with an HGZ extension. This stems from the original creation of the files. The program used to create these files is known as the Vertical Observation Recording Program, or VERREC. The HGZ extension files contain "abstracted and condensed observational data" and the data "are stored in what is called 'binary' format" (National Oceanic and Atmospheric Administration, 1992, pp. 9-1). As explained by NOAA, "this format is efficient for working with the data but it is not appropriate for transferring the data to another computer system" (National Oceanic and Atmospheric Administration, 1992, pp. 9-1). As shown in Figure 2.1, the HGZ files are converted into a Blue Book ASCII file, which is the format used within this project.

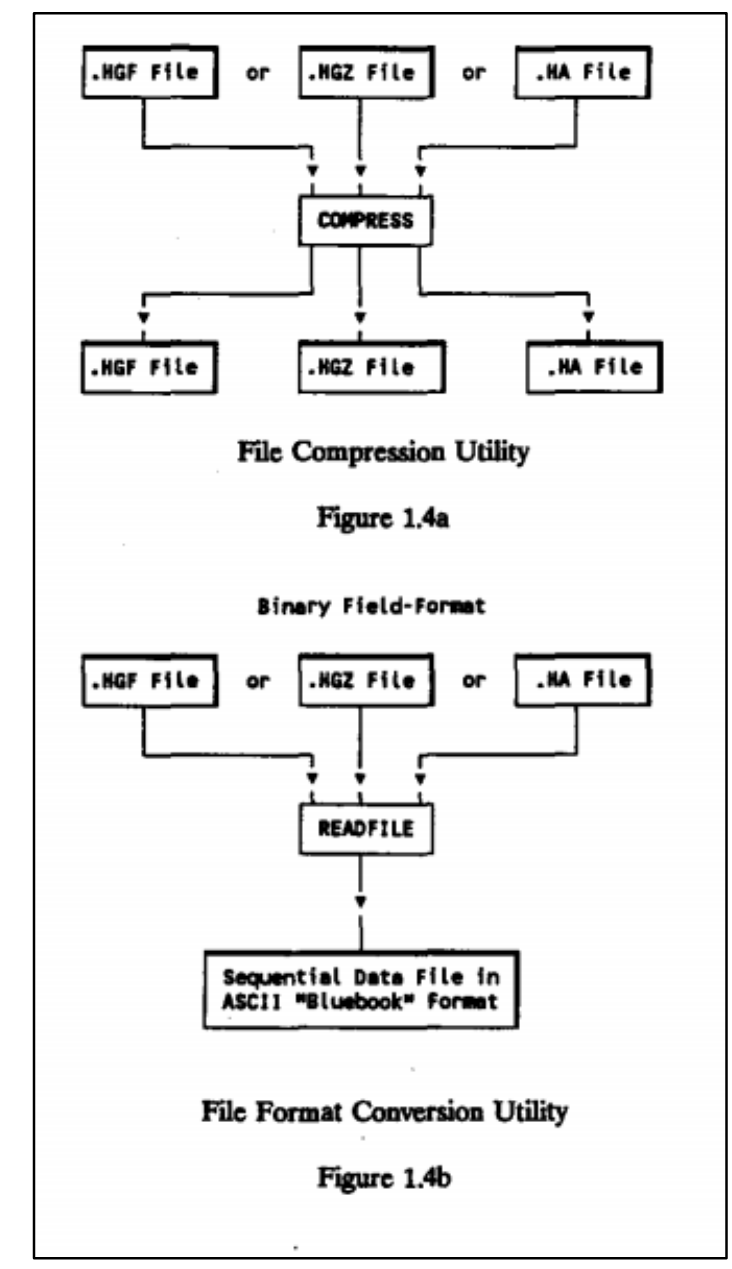

Figure 2.2: HGZ Format Conversion (National Oceanic and Atmospheric Administration, 1992, pp. 9-1) 


\subsection{Summary}

Geodatabase creation has been improved with the newest ArcGIS 10.1 software package, which has simplified the process of dataset management and web application creation. With advancements in technology, the use of ArcGIS for surveyors allows observations to be organized and accessible within a geodatabase.

The following chapters describe the processes used to assist Caltrans with their transition into the use of ArcGIS. The conversion tool allows the client to convert their Blue Book survey observations into an ArcGIS compatible spatial dataset. The analysis tool created allows the client to use the converted dataset and perform temporal analysis quickly and more efficiently than previous methods. 



\section{Chapter 3 -Systems Analysis and Design}

This chapter defines the requirements and workflow for this project in order to clarify the processes used for its completion. The planning of a project takes up the majority of a project's time schedule. Proper planning ensures that all requirements, restrictions, and risks are evaluated before an investment (time, money, etc.) is used for project implementation. This project helps solve the problem of the client, and these requirements outline the solution of the problem.

\subsection{Problem Statement}

The problem of the client was their limited ability to perform spatial and temporal data analyses due to the inefficient storage and organization of their datasets. The client needed an efficient way to convert their text files into spatial information to perform temporal analyses over multiple years of survey observations. The original dataset format did not allow the client to analyze survey observations along the southern California transportation infrastructure efficiently.

\subsection{Requirements Analysis}

The requirements for this project were determined before implementation to reduce redundancy within the workflow of the project. By defining the functional (executable) and non-functional (performance-based) requirements, the scope and scheduling of the project were determined.

The functional requirements for this project cover the executable requirements set by the client. These requirements cover the capabilities required for the deliverable product. The client required the original datasets to be converted and stored within a geodatabase as points and lines. The specific functional requirements were all derived from these basic needs of the client.

The non-functional requirements for this project were based on the performance of the results. These requirements were based on the ArcGIS license held by the client as well as standard specified by NGS. The client held an ArcGIS 10.1 license, so the project contained a geodatabase compatible to this standard. Emails with NGS officials specified that the projection of the geodatabase was held in NAD 83 (2011) in order to match their current geodesic analyses within ArcGIS. Table 1 lists these functional and nonfunctional requirements, along with their ultimate priority within the project. 
Table 1. Functional and Non-Functional Requirements.

\begin{tabular}{|c|l|c|}
\hline $\begin{array}{c}\text { Functional/ } \\
\text { Non-Functional }\end{array}$ & \multicolumn{1}{|c|}{ Description } & Priority \\
\hline Functional & $\begin{array}{l}\text { Transform HGZ files and update geodatabase } \\
\text { tables }\end{array}$ & High \\
\hline Functional & $\begin{array}{l}\text { Query for benchmarks with large temporal } \\
\text { elevation changes }\end{array}$ & High \\
\hline Functional & $\begin{array}{l}\text { From HGZ file create polyline features for } \\
\text { each survey job }\end{array}$ & High \\
\hline Functional & $\begin{array}{l}\text { Conversion tool shall sort fields into } \\
\text { appropriate format (eg. Integer, text, date, } \\
\text { etc.) }\end{array}$ & High \\
\hline Functional & $\begin{array}{l}\text { Manage data in a Geodatabase, organized by } \\
\text { surveyID }\end{array}$ & High \\
\hline Functional & $\begin{array}{l}\text { All survey observations will be maintained } \\
\text { during conversion (no data shall be lost) }\end{array}$ & High \\
\hline Non-Functional & $\begin{array}{l}\text { Geodatabase will store spatial geographic } \\
\text { coordinate system as NAD 83 (2011) for } \\
\text { NGS standard }\end{array}$ & Medium \\
\hline Non-Functional & Requires ArcGIS 10.1 \\
\hline
\end{tabular}

\subsection{System Design}

The system design for this project revolved around the organization of the geodatabase design and automated conversion. In order for temporal analyses to be performed, the geodatabase had to be structured properly to allow comparisons of differential elevation changes over time. This design had to comply with the needs of the client, while maintaining the national standards of National Geodetic Survey (NGS). This project dealt with the client's pilot dataset of nine historical surveys in the southern California region. These files were provided to the client by NGS. Upon completion of this project, the client would be able to add additional surveys provided by NGS.

The final system design was organized and stored within a geodatabase compatible with ArcGIS 10.1. The geodatabase contained not only the organized feature classes and tables containing the survey datasets, but also the toolsets for the conversion and analysis. The conversion tool was designed to serve as the connection between the raw datasets and the spatial datasets within the geodatabase. The client required that the conversion tool should fill/update the geodatabase with survey observations from multiple surveys 
when one or more HGZ files provided to the conversion tool. The tool asks for input files and the location of the pre-made geodatabase schema and outputs an updated geodatabase. Organized by date and the survey's ID, the differential elevations could be used for temporal elevation analysis. Figure 3.1 displays the overall process for the system design for this project.

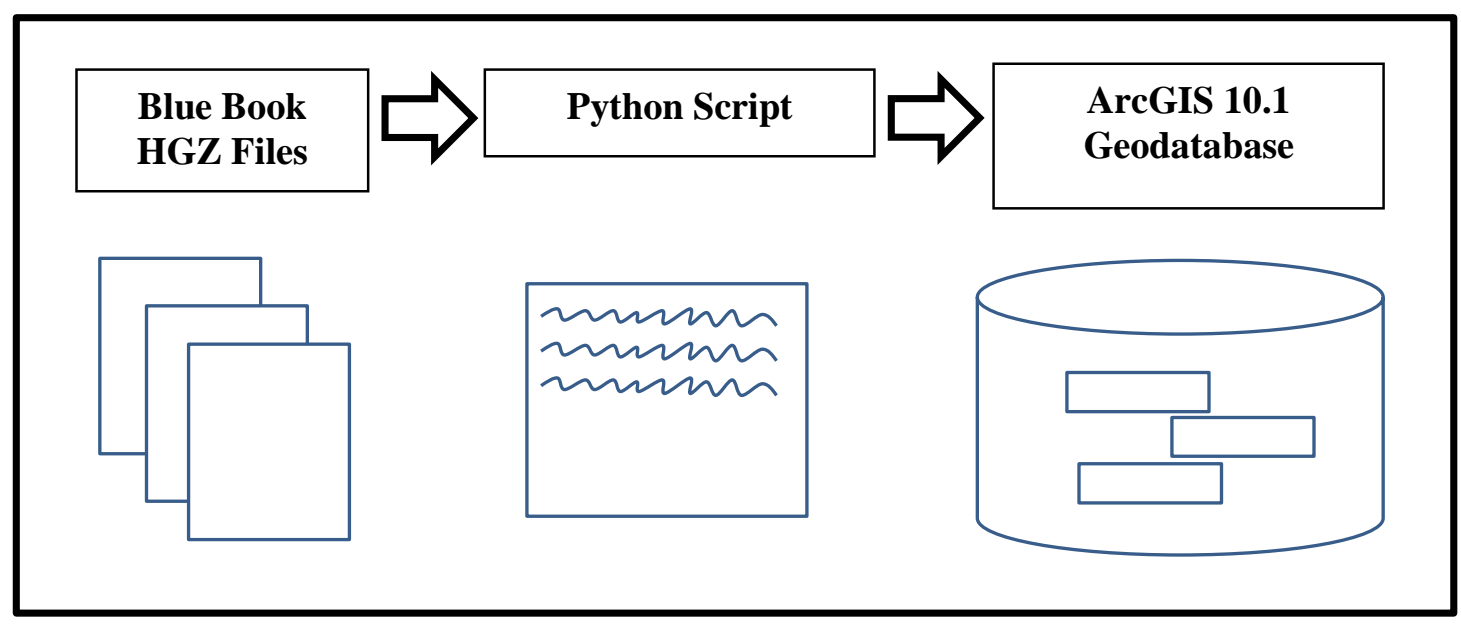

Figure 3.1: Overall System Design Process

Once the survey observations were updated into the geodatabase, consecutive benchmark IDs and date fields were used to compare the differential elevation fields over time. Based on a user-specified threshold of significant differences in height, only repeated runs larger than the threshold were inserted into the results table. This allowed the ArcGIS tool to pinpoint the areas with large elevation changes over the years. The use of ArcGIS technology allows the client to overlay the results with other GIS layers such as geomorphological layers to make decisions regarding the stability of the transportation infrastructure.

\subsection{Project Plan}

Based on the format of their observations, the client was unable to perform any spatial or temporal analyses with the multiple years of information collected. The pilot dataset provided by the client contained surveys dating back to the 1960s. The execution of this project was created in ArcGIS 10.1 and Python to solve the problem of the client.

The first milestone for this project was to determine the requirements necessary for the success of the toolset. Many of these requirements were determined by the client, with consideration of the scope of the overall timeline. The requirements for this project were determined based on how the client needed to use the final result of the tool. The overall plan for the project followed these major steps: requirements analysis, database creation, prototype development and testing, documentation, and deliverables.

This process required constant communications with the client and advising parties to ensure the ending result was efficient and effective, and solved the problem of the client. 


\subsubsection{Requirement Analysis}

To complete the requirement analysis for this project, several meetings were conducted to clarify the needs of the client. During these phone calls, emails, and in-person meetings, the scope was addressed to limit and define the final deliverables in fine detail. Within the time frame of the project completion, the client was available and willing to provide resources and opinions for the details within the database and interface structure. One main setback for the requirements was due to the client not fully understanding the format of the HGZ files. This required additional research and communication with National Geodetic Survey (NGS) personnel.

Once the experts of the Blue Book format clarified the details and provided documentation regarding the HGZ files, the requirements analysis step proceeded toward the creation of a geodatabase schema.

The final requirements created from this process can be referenced in Table 1 earlier in this chapter. Figure 3.2 shows the process of change and approval for the requirements. Once all requirements were settled, the geodatabase schema could be created.

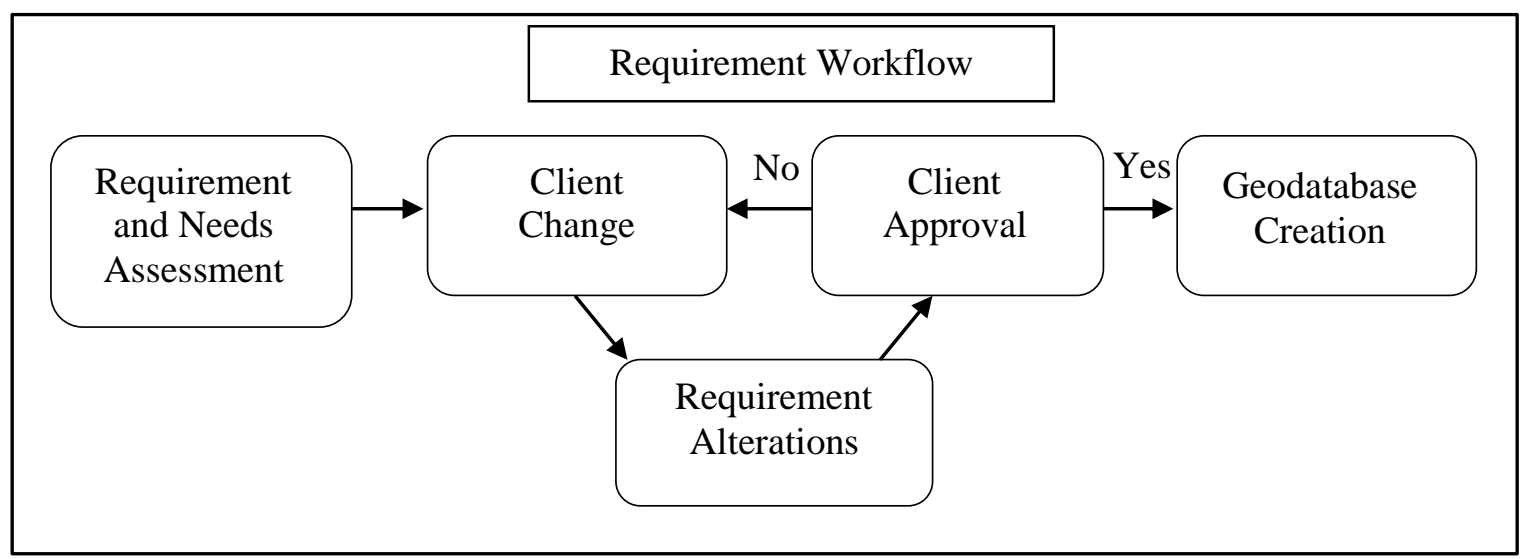

Figure 3.2: Process for Determining Requirements

\subsubsection{Database Creation}

This portion of the project entailed the creation of the database schema for which the HGZ files would be converted into. Database design required the understanding of the analysis which would be performed on the finished product. Many iterations of the geodatabase schema were created until it matched the needs of the client while also storing the HGZ files most efficiently. Understanding the attributes within the HGZ files was necessary in order to efficiently organize these files, which is why the Reduce and Blue Book keys referenced in Chapter 2 were used to create the geodatabase. The client required all information from the HGZ files to be maintained through the conversion process. Figure 3.3 shows the process by which those needs were met. As seen in the figure, once the schema was created, the prototype development and testing began. 


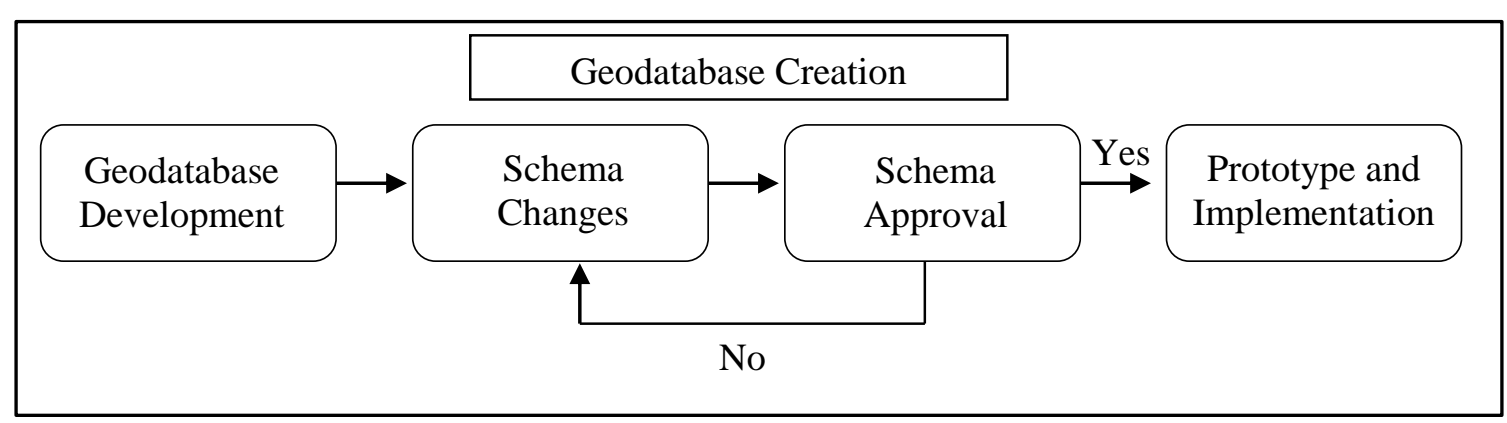

Figure 3.3: Geodatabase Creation Workflow

\subsubsection{Prototype Development and Implementation}

Once a database schema was created for the project, a prototype to convert and store the files was required. The prototype was created and tested with multiple iterations until it worked correctly. Once the prototype worked correctly, it was edited and transformed until it had built-in quality control methods. These quality control methods consist of different methods to handle any differences between the original datasets. These were included to prevent the prototype code from breaking or to ensure that a particular error message would be produced upon faulty use of the conversion tool.

Overall, the prototype conversion tool development relied on various resources for troubleshooting and also a thorough understanding of geodatabase design and structuring within ArcGIS. Upon completion of the prototype, the documentation for the overall project began. Figure 3.4 displays the process used for the prototype design stage.

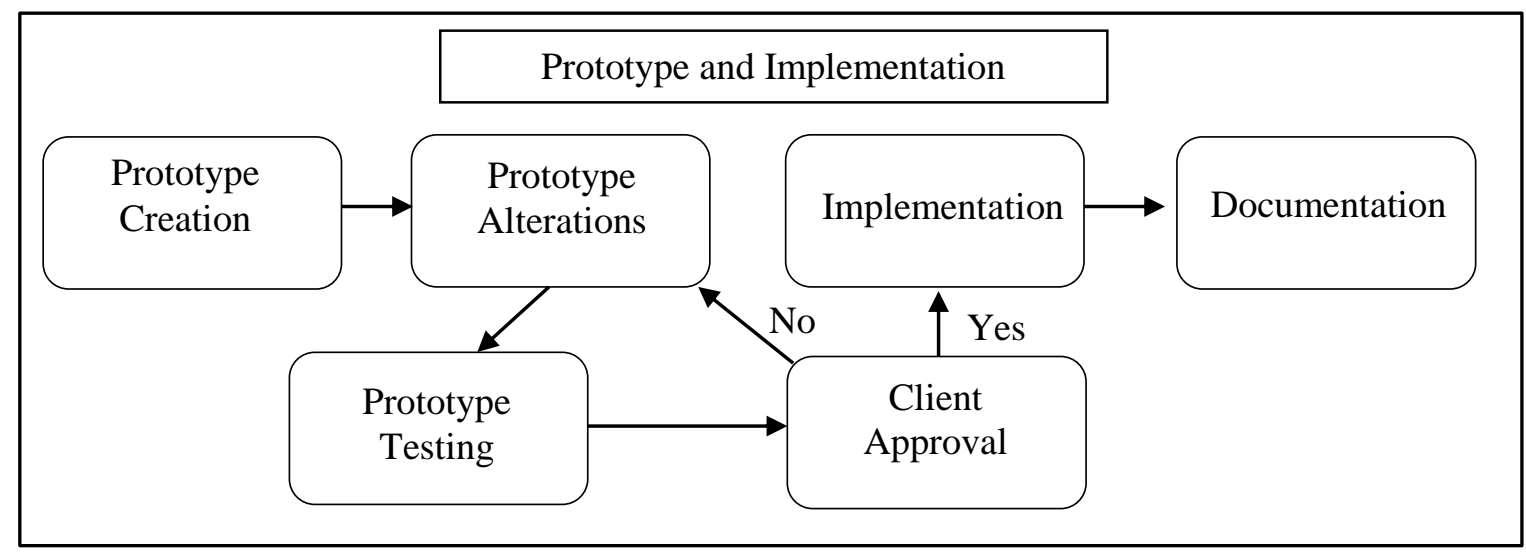

Figure 3.4: Prototype and Implementation Workflow

\subsubsection{Documentation}

The documentation required for this project was a critical factor for the sharing and future use of this project. The client required basic documentation within the Python code and ArcGIS tool interface in order to alter the tool later if necessary. The client also requested a write-up PDF document for the project in order to share the tool to other GIS surveyors 
in the future. This would allow future users to understand the input specifications, the output specifications, and the overall specifications of the tool for any user.

The project also required formal documentation and metadata compilation. This was completed parallel to the implementation of the project and was updated as the project faced new changes. The workflow for the documentation and conclusion of this project is shown in Figure 3.5. This workflow contains the processes required for a Master's degree from the University of Redlands such as the editing and defense milestones.

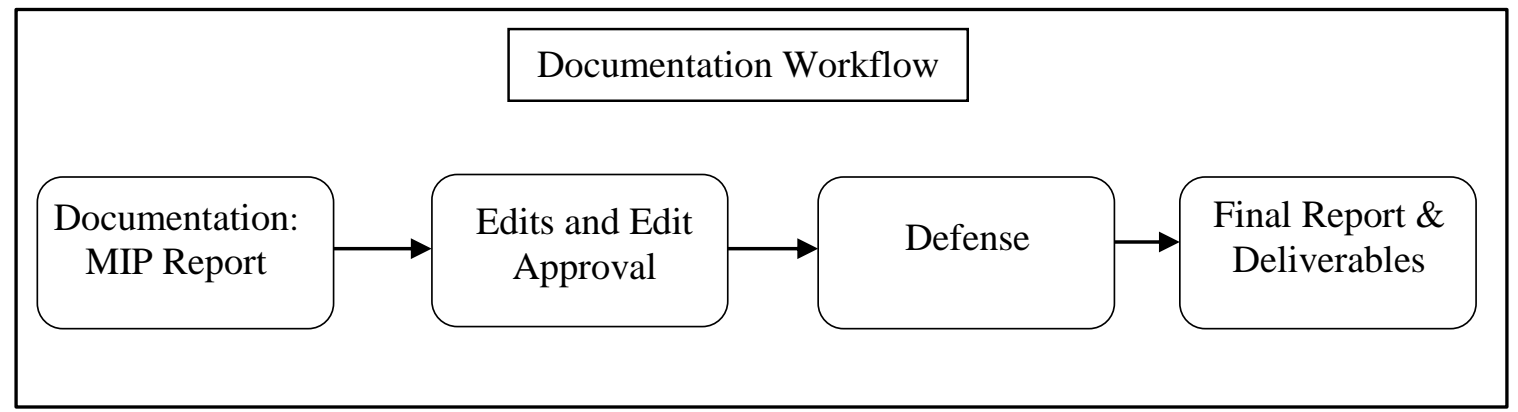

Figure 3.5: Documentation Workflow

\subsubsection{Deliverables}

The main deliverable for this project was an ArcGIS geodatabase. This geodatabase contained the schema for the storage of the converted HGZ survey information, the conversion tool, and the analysis tool. This storage structure streamlined the process for sharing and implementing data conversion. The conversion tool works only with the associated geodatabase, so the packaging of all aspects of this project into the geodatabase was a necessity for the usability of this project. The structure of the geodatabase is explained in detail in Chapter 4.

\subsection{Risk Analysis and Mitigation Planning}

Like any project, this project had risks that had to be considered from the beginning to divert major issues as they appeared. The risks were assessed during the planning for the project and are compiled into Table 2 . 
Table 2. Project Risks and Mitigation Planning

\begin{tabular}{|l|c|c|c|l|}
\hline \multicolumn{1}{|c|}{ Risk } & Severity & Probability & Total Risk & \multicolumn{1}{|c|}{ Mitigation } \\
\hline $\begin{array}{l}\text { Tool Incomplete by } \\
\text { deadline }\end{array}$ & 5 & 1 & 5 & $\begin{array}{l}\text { Use Gantt Chart to } \\
\text { map timeline and set } \\
\text { deadlines with client }\end{array}$ \\
\hline $\begin{array}{l}\text { Requirement } \\
\text { Changes/Scope } \\
\text { Creep }\end{array}$ & 4 & 1 & 4 & $\begin{array}{l}\text { Use due diligence to } \\
\text { create requirements } \\
\text { before } \\
\text { implementation }\end{array}$ \\
\hline $\begin{array}{l}\text { Tool Functionality } \\
\text { Failure }\end{array}$ & 4 & 1 & 4 & $\begin{array}{l}\text { Creating a test } \\
\text { geodatabase before } \\
\text { the creation of the } \\
\text { Python script }\end{array}$ \\
\hline $\begin{array}{l}\text { Inexperience with } \\
\text { Python/Arcpy } \\
\text { scripting }\end{array}$ & 4 & 2 & 8 & $\begin{array}{l}\text { Enrolled in Python } \\
\text { course and Esri } \\
\text { training along with } \\
\text { communication with } \\
\text { Arcpy expert }\end{array}$ \\
\hline
\end{tabular}

A mitigation plan for each risk was created to avoid negative impacts on the project completion. The total risk numeric value provides a magnitude of each risk, computed by multiplying the severity and probability of a risk occurring. The risks considered during the evaluation included timing, scope creep, tool failure, and technology inexperience.

The risk of an incomplete tool depended on the overall timing and lack of time management for this project. A Gantt chart, a timeline for scheduling milestones, was used to mitigate the risk of not completing the toolset. Scope creep refers to the overall goals of a project exceeding the timeline or capabilities of a project. By setting realistic and manageable requirements during the planning stages, the concept of taking on more than can be handled is reduced.

Tool failure refers to the inability of the conversion or temporal analysis tool to work properly using the given datasets. This failure would be due to poor organization of the database and would ultimately require the Python conversion code to be rewritten. To mitigate this risk, the structure of the geodatabase was required to be approved by all parties before the Python scripting could commence. This avoided any issues within the conversion process to complete the process once, rather than multiple time-consuming iterations.

The final risk of inexperience coincides with the learning process of a Master's degree. While many valuable skills are taught, the specific skills for a project such as this require specialization that is not necessarily covered in enough depth. To mitigate the risk of lacking the knowledge required for this project, multiple classes and relationships were established to gain the required skills. The collection of resources used to complete this project included two Python courses, an advisor familiar with Python, and a Redlands Institute Arcpy expert. 
As shown in the risk table, the major risks faced by this project were scope creep and tool creation issues. These risks also became the biggest issues that required mitigation throughout the project completion. Mitigation plans had been set in place to plan and adjust for these risks. Following the risk matrix allowed the project to run smoothly during issues, and provided a plan if the project was at temporary halt.

\subsection{Summary}

This chapter covered the basic structure for this project's overall plan. The initial planning and building of requirements allowed the project implementation to flow smoother and face issues as they arose. The project's requirements from the client were broad, but the project plan provided specific design and structure and narrows those requirements. Once requirements were determined, these were used to help move the prototype and implementation into their next stages. Documentation was addressed to specify the importance of recording the issues and processes used during a project.

This chapter also addressed the importance of risk analysis for a project. Without risk analysis, a project could fail despite thorough planning and scope analysis. The overall workflow for the project completion was introduced in order to help introduce the following chapters, which will elaborate on the design and implementation of the project. 


\section{Chapter 4 -Database Design}

A comprehensive geodatabase is an important key for the development of an efficient GIS project. Understanding the dataset and what analyses will be performed is essential for the creation of a database schema. A conceptual model contains the processes and classes for a database, and the logical model mirrors the organization of these classes. This project required a large amount of data loading and preparation, and relied on a solid geodatabase structure before the conversion process could begin. This chapter describes the two data models and introduces the methods for the data loading, which are discussed in further detail in the following chapter (Chapter 5). This project ultimately allowed the client to convert and utilize their survey data files, but the actual result of the project was automating the process for the client rather than dealing with the data in detail.

\subsection{Conceptual Data Model}

The conceptual model for this project gathered the major components from the input datasets, and organized them into corresponding classes. The model required a thorough knowledge of the surveying techniques used to compile the datasets, and also the consideration of how the final geodatabase would be used. While the datasets from the client contained a vast amount of information, the overall organization of the data was relatively basic. Figure 4.1 presents the overall conceptual model used to organize the data structure.

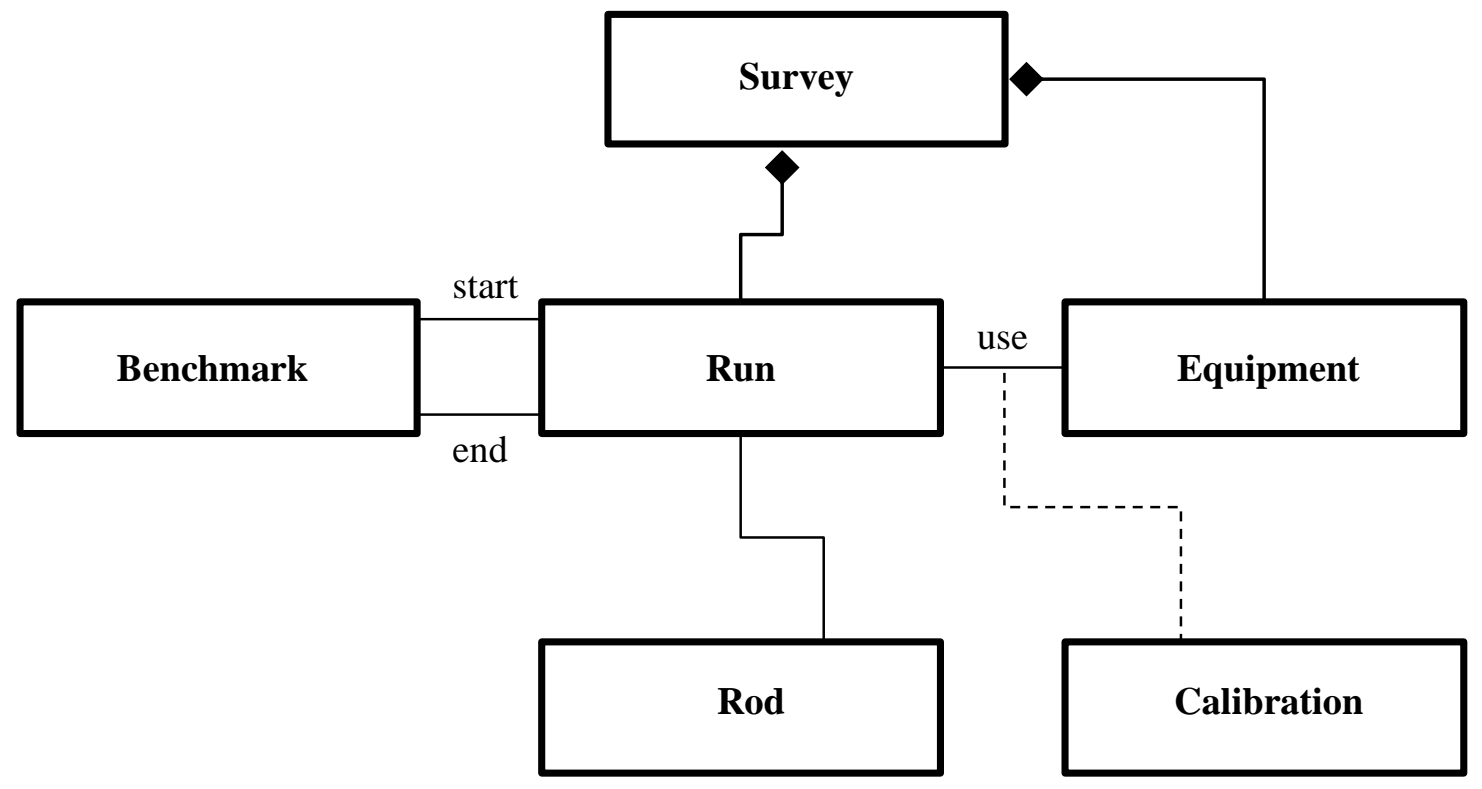

Figure 4.1: Conceptual Model for the Dataset

The model displays the main components of the datasets all contained within a single survey. The raw survey observations from a HGZ text file were contained within the runs, while some adjusted measurements and heights were all stored within the 
benchmark records. The runs start and end with benchmark points, which contain the geographical coordinates of the measurements. All measurements were recorded with equipment, which required calibration. Different rods were used for different runs within the surveys.

The data within the text files could be utilized by the client in order to perform corrections and calculations. This could all be performed using the resulting geodatabase. The client ultimately needed to perform regression analysis on the values within the dataset, but that is beyond the scope of this project. This project allows the client to use the datasets they have, and gives them the capability to analyze them by organizing their databases efficiently.

\subsection{Logical Data Model}

The logical data model consists of the database organization comprised from the original text file datasets. This organization utilized the attributes provided within the records of each survey and allowed spatial analyses to be performed on the resulting ArcGIS feature classes. The client required certain information to be stored within point and line feature classes, while non-spatial data were placed into tables which could be joined with the spatial feature classes. The main spatial feature classes were Benchmarks as point features and Runs as line features. The overall structure of this organization is shown in Figure 4.2. The details of each feature class and table are explained in the following sections. 


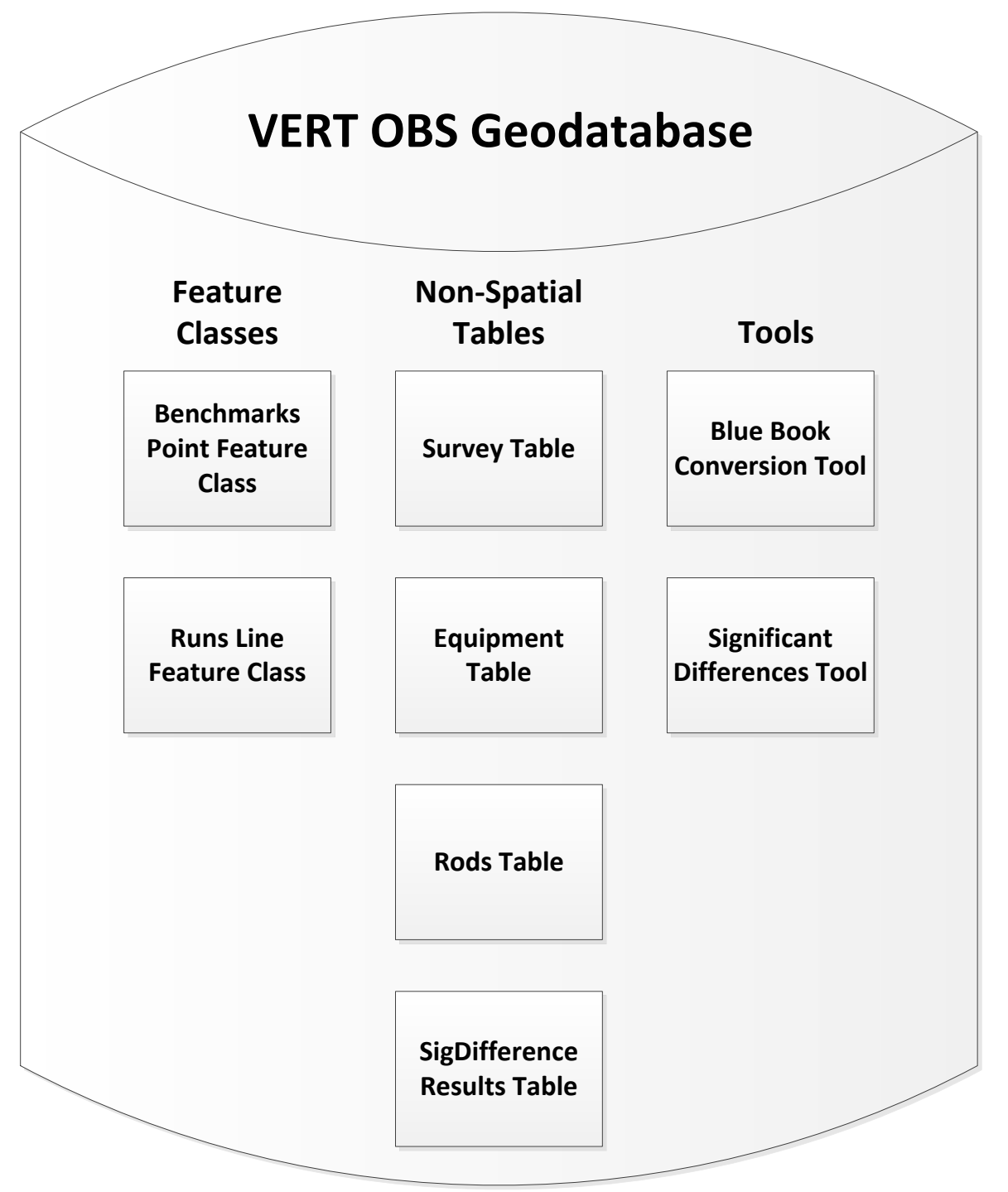

Figure 4.2: Basic Structure of Geodatabase

All tables and feature classes within the geodatabase required key fields in order for any inter-tabular analysis to be completed. The main key field between all tables was the specific survey identification (SurveyID) that differentiated each survey from others within a table. The key between the survey datasets and the analysis tool result table is the ObjectID from a run with significant height change. This links the SigDifference table to the Runs table to visualize the results. The organization of the tables within the database maintain third order form and allow for different key values to be used depending on the analysis that needs to be completed.

\subsubsection{Benchmarks Point Feature Class}

The differential leveling surveys used by the client require measurements to be made from long-term stable points known as benchmarks. As explained in Chapter 2, these 
points are stationary and used as reference points for differential leveling measurements. Within each survey, the benchmarks are recorded with a latitude and longitude, along with several other attributes. Every benchmark was recorded using a unique identifier recognized by NGS as a Point Identification (PID) and a unique identifier recognized by a particular survey known as an Station Serial Number (SSN). In order to differentiate between what benchmarks were included within each survey, an additional field "SurveyID" was included in the feature class table in addition to the original attributes from the input text file.

In order to make the main key fields maintain first order form, the data type for these fields had to be the same throughout the geodatabase. These fields were: SurveyID, PID, SSN, ObjectID, and all Date fields. An example of some of the Benchmark fields and their data types are shown in Figure 4.3.

\begin{tabular}{|l|l|}
\hline \multicolumn{1}{|c|}{ Field Name } & \multicolumn{1}{c|}{ Data Type } \\
\hline OBJECTID & Object ID \\
\hline SHAPE & Geometry \\
\hline SurveyID & Text \\
\hline JobCode30 & Text \\
\hline PID & Text \\
\hline SSN & Long Integer \\
\hline Latitude_DD & Float \\
\hline Latitude_DMS & Text \\
\hline Latitude_Hem & Text \\
\hline Longitude_DD & Float \\
\hline Longitude_DMS & Text \\
\hline Longitude_Hem & Text \\
\hline Position & Text \\
\hline
\end{tabular}

Figure 4.3: Example of Benchmark Field and Field Data Type

\subsubsection{Runs Line Feature Class}

The raw observations within a survey data file were the differential height measurements taken between two benchmark points. Because these measurements included a running length, the distance between two benchmarks, they were individually represented as a line segment. Similarly to the benchmarks, a "SurveyID" field was included in the feature class to recognize which runs belonged to which particular survey.

Due to the request of the client to enable the geodatabase to be used for temporal analysis, the Runs feature class included the date and time fields. This allowed for changes of differential height to be recognized over time. These had to be created within the conversion tool to match an ArcGIS compatible Date field format. Figure 4.4 shows an example of some of the fields and their data types. 


\begin{tabular}{||l|l|}
\hline \multicolumn{1}{|c|}{ Field Name } & \multicolumn{1}{c|}{ Data Type } \\
\hline OBJECTID & Object ID \\
\hline SHAPE & Geometry \\
\hline SurveyID & Text \\
\hline JobCode41 & Text \\
\hline DateRun41 & Text \\
\hline StartSSN & Long Integer \\
\hline StartPID & Text \\
\hline EndSSN & Long Integer \\
\hline EndPID & Text \\
\hline TimeCode & Text \\
\hline StartTime41 & Text \\
\hline StartDateTime & Date \\
\hline EndTime & Text \\
\hline
\end{tabular}

Figure 4.4: Example of Runs Field and Data Types

\subsubsection{Non-Spatial Tables}

Information from the original files that were not correlated directly to any spatial location within the feature class data were organized within non-spatial tables in the geodatabase. These non-spatial tables were as follows: Rods, Equipment, Survey, and SigDifference. The Equipment and Survey tables were filled using the client's pilot dataset, while the Rods table was used as a placeholder for any files from the client which might include the optional rod information job codes. The SigDifference table contained the output results from the analysis tool. The tables provided the client with the capability to use input text files which include more detailed information than the pilot dataset. The non-spatial tables also allowed the tool to maintain all information from the original files as required by the client. Some of the information within these files remained in text format since the fields did not require analyses performed on the values, such as the basic information regarding a particular survey. Figure 4.5 shows an example of some of these table data types and field names.

\begin{tabular}{||l|l|}
\hline \multicolumn{1}{|c|}{ Field Name } & \multicolumn{1}{c|}{ Data Type } \\
\hline OBJECTID & Object ID \\
\hline JobCodeAA & Text \\
\hline Vertobs & Text \\
\hline AgencyAbrev & Text \\
\hline Agency & Text \\
\hline DateCreated & Text \\
\hline Version & Text \\
\hline Datum & Text \\
\hline JobCode10 & Text \\
\hline AccessionPre & Text \\
\hline AccessionNum & Text \\
\hline SurveylD & Text \\
\hline LinePart & Text \\
\hline
\end{tabular}

Figure 4.5: Example of Survey Field and Data Types 
For the analysis tool, the output consisted of a non-spatial table within the geodatabase. When the tool runs, the ObjectID for one of the matching runs is recorded into the table to show the spatial location of the run. This table could be joined with the Runs feature class based on the ObjectID field, and could therefore allow the client to spatially represent the regions of major height changes and overlay the results with other layers.

Overall, the geodatabase contained the feature classes, tables, and tools created within this project, and allowed the client to access all of the resources from these surveys in one location. When any new surveys needed to be input, the conversion tool could add the dataset to the geodatabase at any time. The overall structure for this project's geodatabase is shown in Figure 4.6.

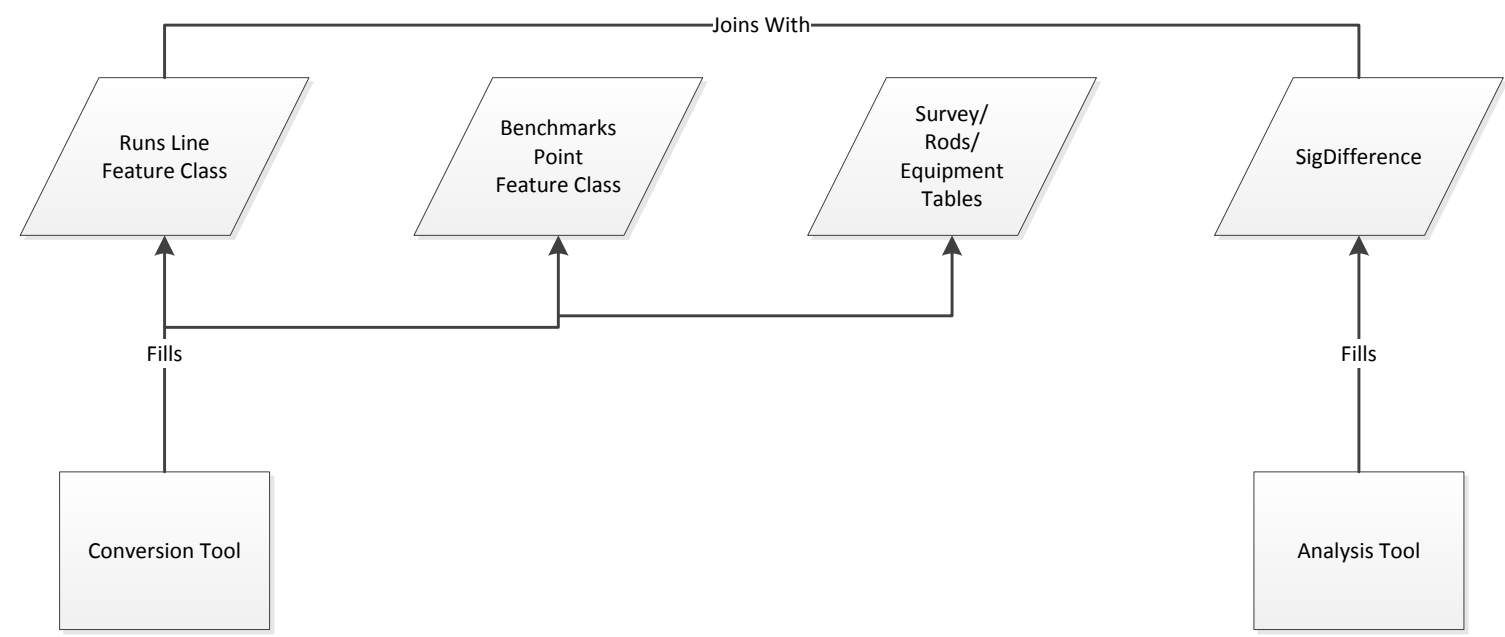

Figure 4.6: Overall Relationship of Geodatabase Tables and Tools

\subsection{Data Sources}

The HGZ text files containing all survey observations came from Caltrans, who ultimately received them from NGS. The format used by NGS for these surveys follows the Blue Book format described in Chapter 2. While this format is used nationwide, this project only used a pilot dataset of this format used by Caltrans. This sample consisted of nine separate surveys completed in the southern California region, and all align closely with transportation infrastructure. This allowed the results of this project to be used for transportation infrastructure analysis.

The metadata for this project was fully based on the Blue Book and Reduce key PDFs explained in Chapter 2. This document specified the meanings of each line record and the attributes associated with different spaces within each line. Based on the needs of the client, the database design was structured to align with their future use of the converted dataset. 


\subsection{Data Collection Methods}

The client provided nine sample HGZ files for this project to test the concept of conversion. As stated in Chapter 2, this Blue Book format dictated the process for data storage. Surveyors collected the differential leveling measurements during one survey at a time using surveying rods and equipment. These surveys were then converted into computers to store the information into a digital format. This digital format is the Blue Book format used for this project.

For this project, there was no data collection other than collecting the datasets from the client and understanding the format. The Blue Book and Reduce format key PDFs specified the types of data which would be contained within the text files the client could encounter.

\subsection{Data Scrubbing and Loading}

This project relied heavily on the scrubbing and loading of the raw dataset into a geodatabase to perform spatial analysis. While the PDF keys to the dataset specified the positions within each line, there were inconsistencies among datasets. Examples of these inconsistencies were values with extra spaces or outlier observations due to human error. These differences were handled within the Python script to control the quality of each dataset.

The HGZ files were loaded using an ArcGIS user interface and a linked Python script which ran through each line of every survey. Each line of the data contained a job code, which specified what attributes were contained within the line data. The Python script differentiated each line based on the job code in its leading position, and parsed the data appropriately to separate the attributes. The parsing required the Blue Book key to define the proper attributes within each job code for each attribute.

Once each line's job code was specified, the line could be separated into its various fields. All attributes and fields within the HGZ datasets had to match their corresponding field types within the geodatabase, which required the conversion of certain fields. The different lines within the files contained variations of spacing and missing attributes, which had to be handled within the Python script.

Conversion into proper field data types was necessary in order to convert the text files into spatial datasets. Once attributes were parsed and converted to the proper data types, they were updated into the proper database table or feature class.

The full process for this Python coding and conversion is described in full within Chapter 5.

\subsection{Summary}

While the client provided concise and complete pilot datasets for the conversion tool, each file required a certain extent of data preparation to create ArcGIS compatible datasets. Understanding the client's datasets and how they would be processed later was required in order to organize the conceptual and logical data models for this project. Bluebook surveying datasets such as the pilot dataset provided by the client require temporal and spatial analysis to help make better choices for their company, which requires a solid database structure. A solid database structure allowed the client's data to 
be analyzed in an organized and intuitive setting. This chapter described the process that was used to organize the datasets for the client. 


\section{Chapter 5 -Implementation}

The implementation for this project consisted of the completion of many steps that relied on preceding steps in order to move forward. This waterfall project execution method relied on the requirements and implementation of the project to align to move forward. The methodologies and workflow for the project are described within this chapter to illustrate the processes used to complete the project for the client. This chapter justifies the use of the ArcGIS geodatabase data organization method, and also relays the use of Python for the conversion process.

Once the text files were converted into spatial information, a tool for temporal analysis allowed the client to use any dataset created from the conversion tool. The steps towards the completion of the analysis toolset are described in this chapter.

\subsection{Geodatabase Creation}

The ArcGIS 10.1 geodatabase structure was used for this project to integrate the workflow of the client. The organizational structure of the dataset required the survey observations to be stored into multiple tables as well as point and line features. The geodatabase schema was created within ArcGIS to define the locations and properties of the database structure before loading the dataset. An Esri File Geodatabase was chosen for this project to streamline the conversion and analysis process for the client. The client maintained an ArcGIS 10.1 license, so a File Geodatabase was ideal for the client because it was compatible and transferable as a final product.

The schema for the geodatabase was the first step toward creating a conversion tool for the client. By creating the geodatabase, the conversion tool would be capable of filling the tables automatically. Creating the database also enabled the easy transfer of the schema and tools to the client. The field types for the different feature classes and tables were created during the schema creation.

\subsection{Basic Dataset Prepping Using Python}

The ArcGIS platform works directly with the Python coding language, which allows for ArcGIS functionality to be used within a Python code. Python scripts allow for the integration of ArcGIS software with personalized functions. While the ArcGIS ModelBuilder allows for customized functions, the level of detail required for this project required Python coding to read through the HGZ files and handle exceptions for differences among the files. For every difference among HGZ files, Python coding could handle the different operations and possibilities for the format of the dataset as described in the last section.

The HGZ files contained a relatively specific pattern throughout the files. Although the pilot dataset contained the same job codes throughout, the Blue Book key showed the possibility of additional job codes appearing in the NGS format. While there was a consistent pattern, each file contained specific data that had to be managed before any conversion methods could be used. Figure 5.1 shows an example of part of an HGZ file in its raw form within Notepad ++ . The figure shows the dominating pattern of 
differences among some of the line data, but that the first three positions a the line were job codes. Each job code defined a different pattern for that particular line of data. Each job code defines specific attributes in particular positions within the line, which could be handled within Python using line indexing.

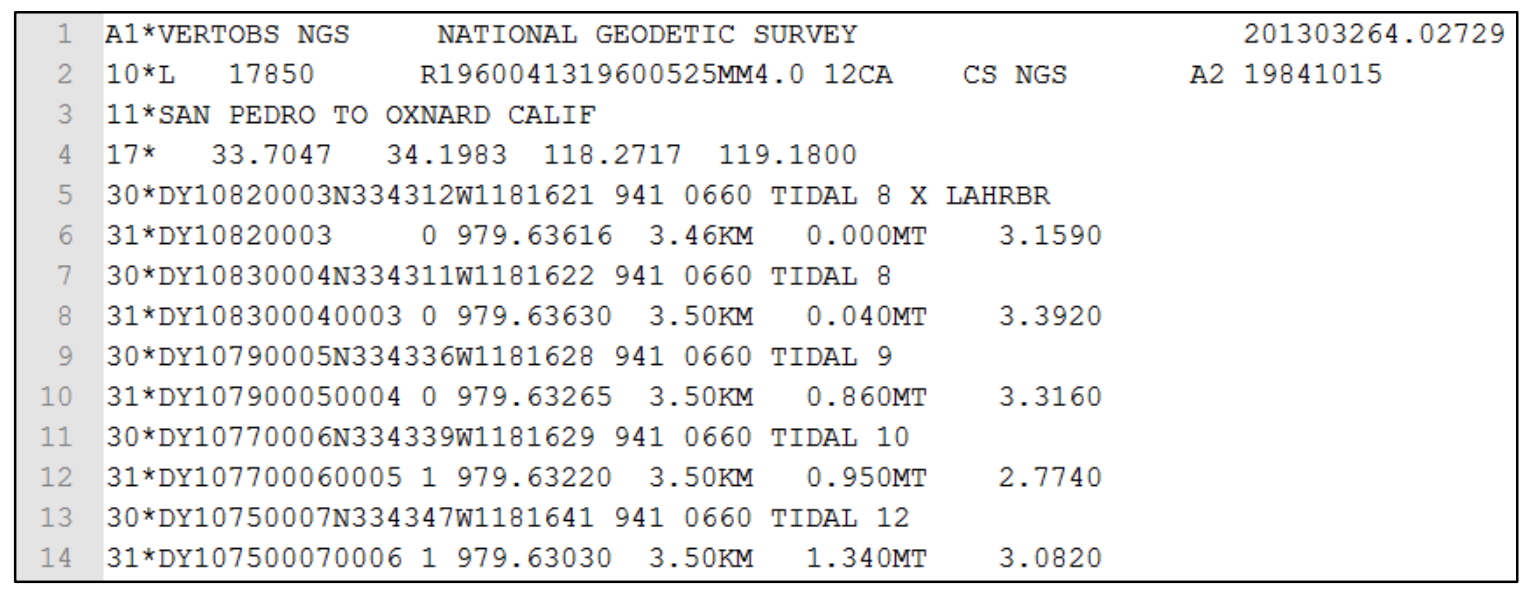

Figure 5.1: Example of Raw Data

The first step within Python was to read the input HGZ file. The ability to batch process multiple survey files required a section of the code to read through each survey one-by-one. A different line within the Python script allowed the tool to read each input HGZ file line-by-line. By following the Blue Book key described in earlier chapters, each line position could be parsed based on its job code. This was completed using conditional statements to determine what information was stored within a particular line. Depending on the first two positions of a line, the script would enter a new section of code which assigned each partition of a line to its appropriate attribute name and type. The steps of this separation and conditional process are shown in Figure 5.2.

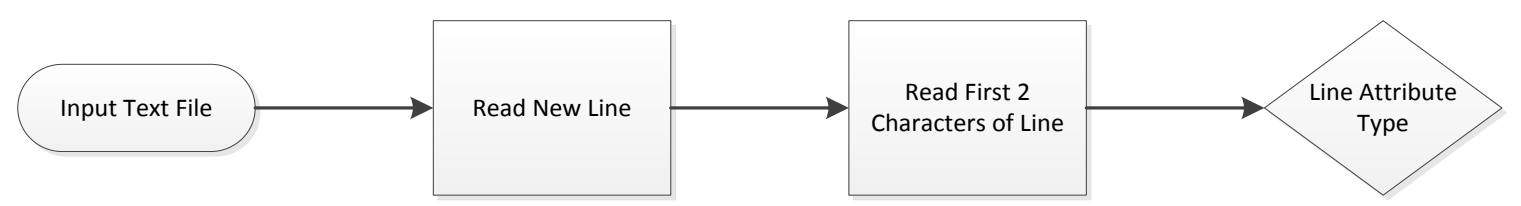

Figure 5.2: Handling Different Features Based on Line Record Type

Once each line was capable of being handled on its own, the indexing for each attribute within a line had to be handled. While the PDF key specified the positions which an attribute would fall within a line, sometimes the indexing would include extra spaces or no attribute value. To handle the spacing issues, a function was used to remove any spaces at the beginning or end of the indexed position. Figure 5.3 shows the process of indexing and use of the "strip" function within Python to remove extra spaces within each parse of the line data. 


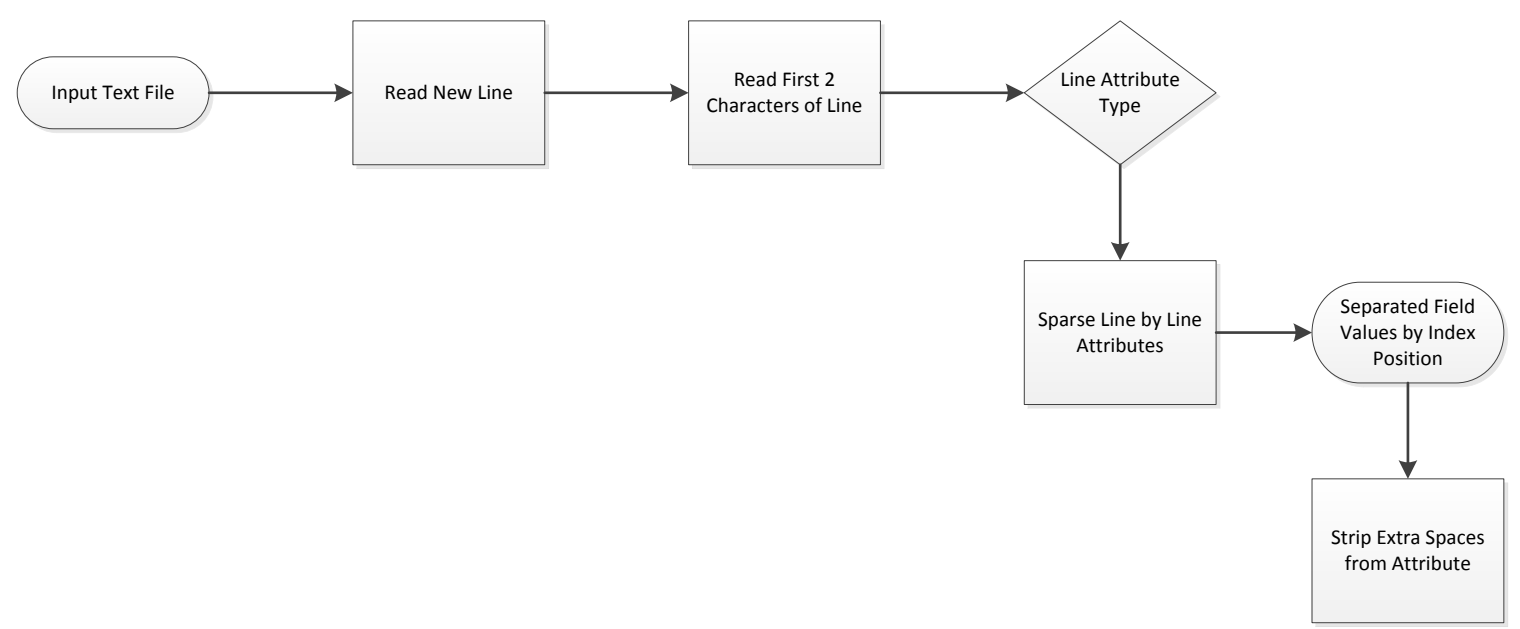

Figure 5.3: Parsing and Stripping the Raw Data

Another discrepancy within the dataset was the differing line endings. While the key specified that a line could end after a certain position, the line might actually end before the specified position. Every line ended with an escape value of ' $\mathrm{n}$ ' which could not be processed by the Python code into usable data. Since this could potentially occur in any line position after the job code, a "replace" function was used within every parsing to handle the escape value in any position. The built-in replacement Python function replaced a specified value with a different specified value. For these escape values, the "In" was replaced with a blank value "". Figure 5.4 shows the process of the line indexing, and replacement of "In" with an empty position "'".

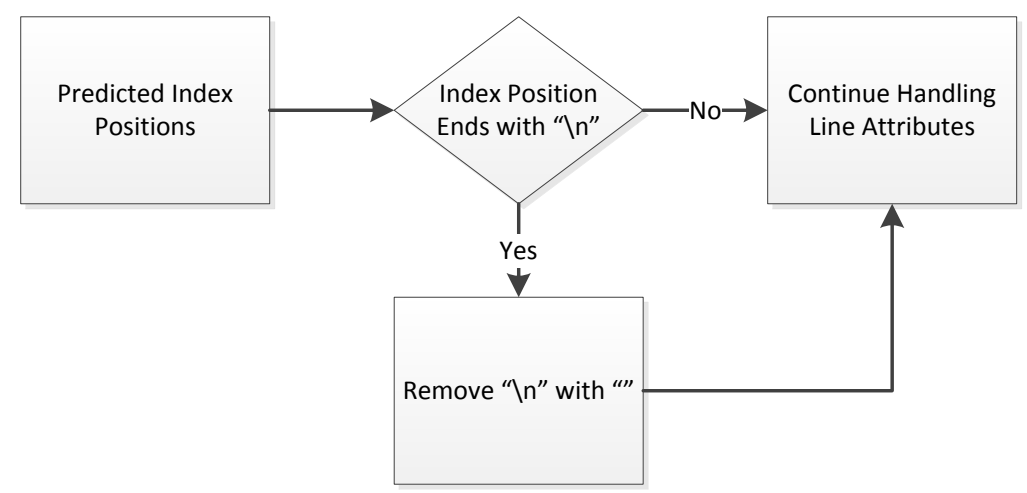

Figure 5.4: Line Ending Clean-up methods

Once basic data preparation was completed for all job codes, the Python code still had to convert the line data into temporal, spatial, or integer values.

\subsection{Handling Field Attributes Within Python}

Python allowed this project to handle multiple versions of the Blue Book formatted files. To create spatial data, the text files had to contain spatial features. The files provided by the client contained coordinates for the benchmark points, along with a unique SSN and PID to identify the start and ending survey points for a line segment. The formatting for 
the coordinates was provided as degrees, minutes, and seconds with no separations, so these had to be transformed in order for ArcMap to recognize them as points on a map. A conversion function was written to store the degrees, minutes, and seconds separately, and convert them into a decimal degrees format. Figure 5.5 shows the process used to convert the data into spatial information. This process created a decimal degree format which could be used by ArcMap to store the dataset as spatial features.

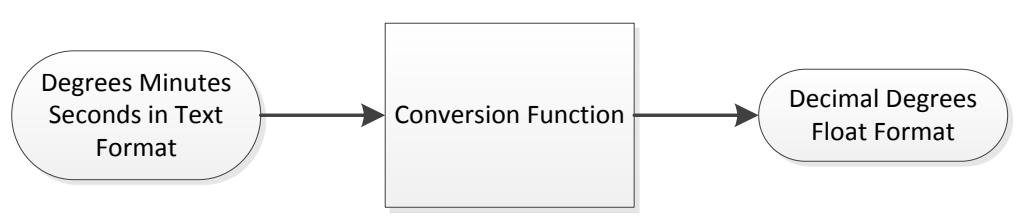

Figure 5.5: Converting DMS to DD

Once the coordinates were in a spatial format, other attributes within different lines had to be handled with special data types apart from the original text formatting. Fields that required this conversion used integer or float conversion methods to transform them into proper fields which could be analyzed by the client within ArcMap. All field types within the Python code were required to match the field type within the ArcGIS geodatabase schema in order for the conversion process to work, so a set of checks were incorporated in order to handle exceptions within the data. Python conditional statements allowed for null values to be handled. For example, if a value was an integer field, but a particular dataset did not include a value for that field, no value would be added to the table. This would result in a $<$ Null $>$ value appearing in a row for that field. This was done because an empty string is not recognized by ArcPy as an integer field. The conditional statements kept the code from failing. Figure 5.6 shows an example of this process.

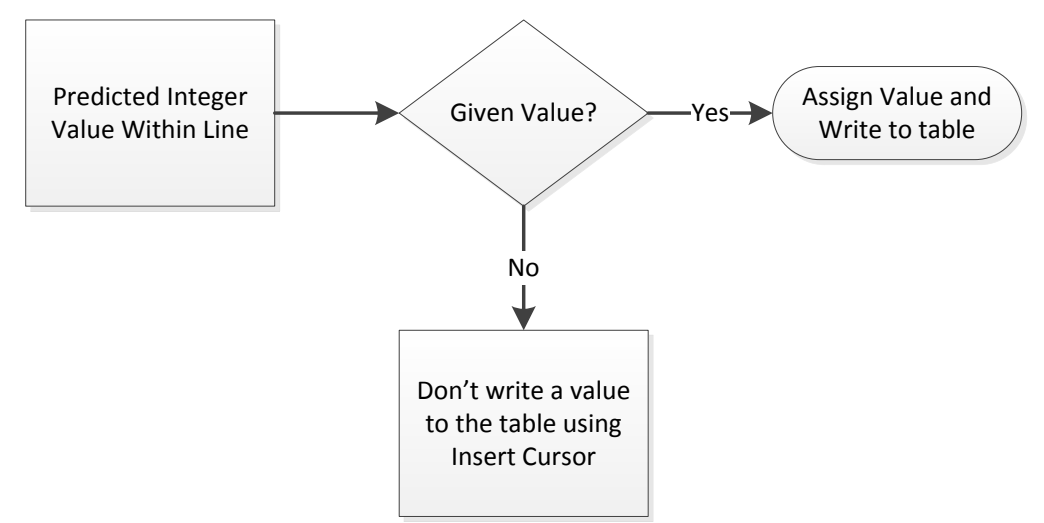

Figure 5.6: Handling Null Values for Integer Fields

The final step for preparing the dataset was to create compatible date and time fields. ArcGIS recognizes certain string formats for their date fields such as YYYY/MM/DD (Year, Month, Day), so a script function was created to format the date fields into a format ArcMap would recognize as a time field. This format could remain as a text field and be recognized by ArcMap, but an additional conversion function created a date/time format. This additional function used the raw date and time of a measurement to allow 
the client to incorporate the raw observation values with the rest of the measurements. This would allow the client to make any necessary adjustments or corrections using survey specifications. Figure 5.7 shows the basic process for both date conversion types.

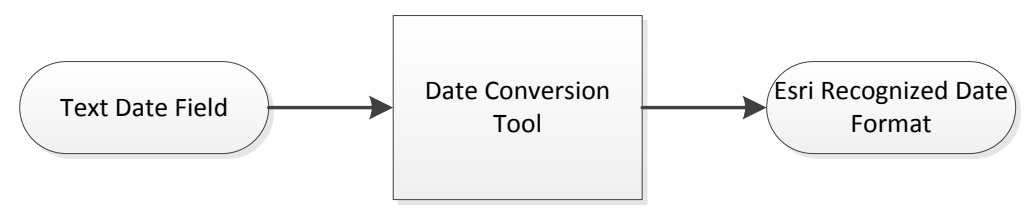

Figure 5.7: Date Field Conversion

Once the data was prepared, the geodatabase loading process could begin using Arcpy methods. The following sections describe the details for this process.

\subsection{Geodatabase Loading using Python}

Once the Python codes handled the data preparation, the field attributes had to be inserted into the geodatabase schema explained in Chapter 4. Once the Python code converted the field type to match the geodatabase, the code had to store the appropriate values for each line record destined for a specific table within the geodatabase. The method used for this process was the Arcpy Insert Cursor and a Python dictionary or list to store the information shared between records.

Due to the organization of the datasets, different line records were inserted into varying tables based on their job codes. Each table or feature class required an Insert Cursor to add rows of data, but the use of multiple cursors required the code to start and end an edit session on the geodatabase. An edit session start and end within the code allowed the whole database to be modified as each survey was added without locking the geodatabase. Each Insert Cursor matched the fields for the table or feature class.

When entering a new section of the code which needed to be placed within a row, a new empty row was first created within ArcPy. The different values of a job code were then stored as variables depending on the parsing of a line, and were assigned to the empty row based on what field they would fill. A dictionary contained a key and a corresponding value, in order to retrieve matching information in other job codes. Dictionaries allowed values to be retrieved by using the key value as a unique identifier. This is how a beginning and ending latitude and longitude were retrieved from the benchmark information to write the Runs line features.

If an Insert Cursor contained multiple job code values within one row of the cursor, the same variable to store the information for a new row had to be used. Since the order of the job codes was specified within the Blue Book standard, the Python code conditional statement processed them in the same order. For example, two job codes for the same row came in pairs. This allowed the Insert Cursor to write one row at a time without the need of storing extra information. When all variables within the job codes were the proper converted format and assigned to the Insert Cursor field values, the Insert Cursor would write a row into the table. This process is shown in Figure 5.8. 


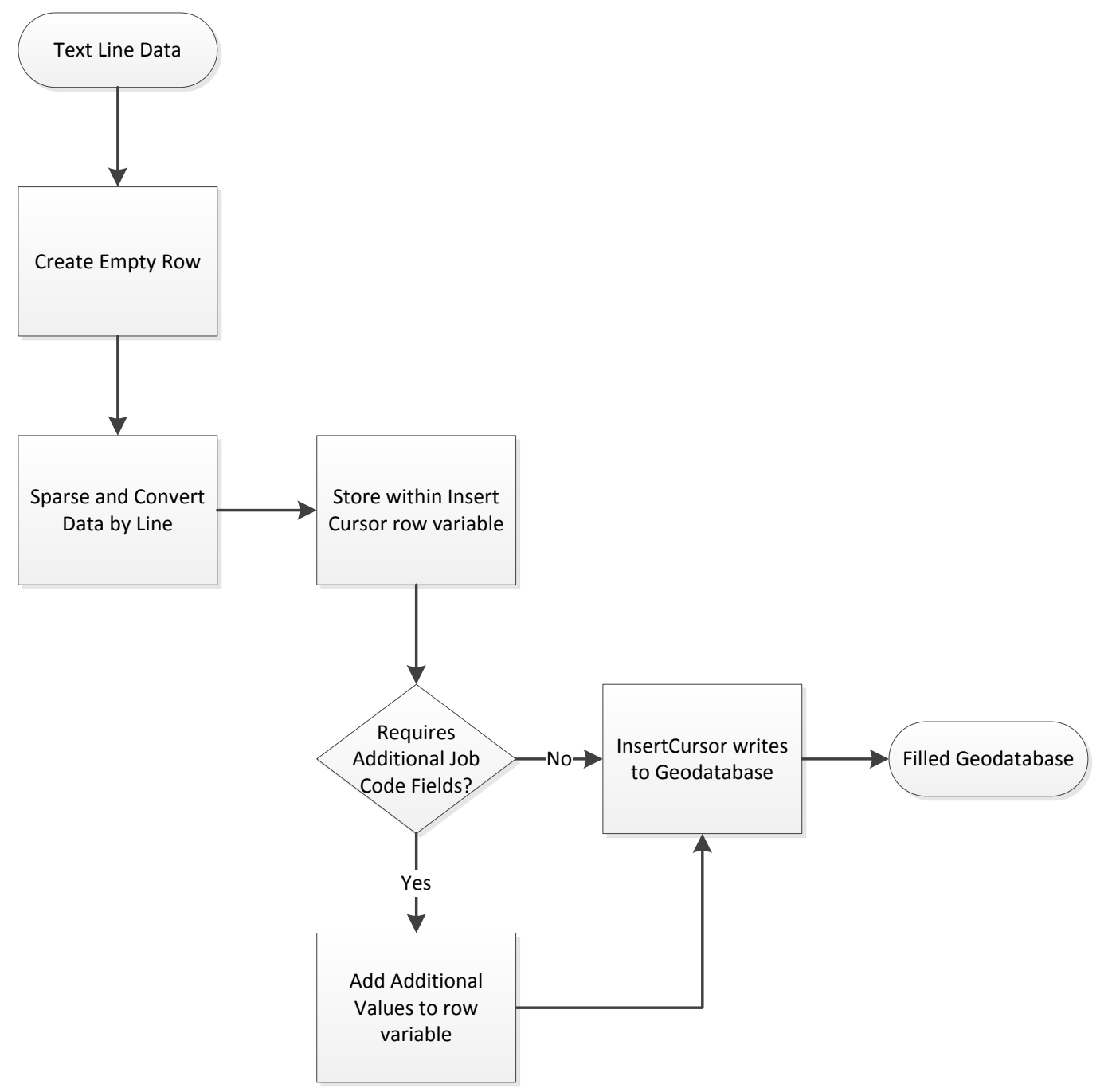

Figure 5.8: Inserting Line Data to Geodatabase

The InsertCursor method was used for both the spatial feature classes and the nonspatial tables. The spatial feature classes included a field which specified the Shape attribute for each row. This method filled the geodatabase as the tool ran through the file, so the tables contained all the information from the surveys once the tool was run. This maintained all values from the original files, as specified by the client.

The feature classes described in the database structure chapter required special coding within Python to create ArcGIS compatible geometries. Because the client's final goal was to perform temporal analysis, these geometries also required timestamps to allow for temporal analysis.

Benchmarks are stationary point locations which a surveyor references to measure elevation differences. These had to be converted from text latitude and longitude numbers, into ArcGIS point features. The point feature class was added to the geodatabase schema described in Chapter 4. The Insert Cursor added a field which held the ArcGIS specified shape specification for points. As a latitude and longitude appeared within the line record for benchmarks, the point was stored as a variable. When the Insert 
Cursor added a row to the point feature class, a spatial benchmark point feature was created for each particular benchmark.

The input HGZ files contained the raw data for the runs directly after all benchmark points for a survey were specified. The raw observations record the start and ending SSN values for a particular run. Since the runs were stored after the benchmark SSN values, the Python code could retrieve these values as starting and ending points for line segments. The value of each point was retrieved by using the SSN number, which was the key within the benchmark line record dictionary described in the previous section. By retrieving the value of the start and ending coordinates from these dictionaries, a line segment was created for each run. By using a line shape specification for the "Runs" Insert Cursor, each row within the feature class contained a particular line segment, with the specified start and end SSN.

\subsection{Handling Repeated Surveys and Non Blue Book Files}

In order for the tool to run correctly, the proper file type had to be used as inputs within the tool. Otherwise, the tool would fail to run and the conversion would not work. To recognize if an improper input was used, an error message was created to warn the user that their inputs were incorrect. In addition, if an HGZ file was drastically different from the pilot set provided by the client, then the tool may not include additional information stored within those files because it would not know how to process the lines. Error messages inform the user if these errors occur, and are recognized by scripts within the Python code.

Another method factor for maintaining the integrity of the geodatabase was to check for redundancy of surveys. As the code read through a survey, if the Survey ID was already within the database, the code would jump to the next survey file and an error message would inform the user that the survey has already been input into the database.

\subsection{Temporal Analysis Toolset}

In order to prove the validity of the conversion tool and geodatabase layout, a temporal analysis toolset was created to pinpoint areas of significant height change between surveys. The tool was created to perform the following tasks:

- Recognize line segments repeated between surveys;

- Calculate the difference between the differential leveling of repeated line segments;

- Compare the difference between a user-specified "significant" value;

The tool ultimately retrieved the raw observations that exceed the user-specified threshold and placed them in a table for additional analyses. This method was used in order for the client to use the raw data and perform any additional analyses on the information. This was done as opposed to creating a table of calculated values because surveyors perform calculations from raw data to keep the integrity of the raw observations.

The analysis tool, like the conversion tool, was created within a Python script and linked to an ArcGIS tool interface. Python was used to iterate through the elevation values and write analyses results to the SigDifference table described in Chapter 4. 


\subsubsection{Recognizing Similar Line Segments}

In order to pinpoint significant height changes over time and space, the first step was to locate similar runs between surveys. Each survey measured elevation changes between particular benchmarks, but the exact order of the runs were not identical between surveys. As explained in chapter 4, the Runs feature class stored the line features representing the runs between two benchmarks. The Runs feature class also stored the raw observation measurements, including the differential leveling elevation measurement. In order to compare the differential leveling values between surveys, the analysis tool had to first locate runs within different surveys between the same two benchmarks.

To locate similar runs between surveys, the Runs attribute table was conceptually joined with itself using two Search Cursors commands. A Search Cursor reads through the rows of a table and can return values within the table. This process compared the table with itself. First, a cursor was created to run through the table and examine the values of the start and end benchmark values. As the cursor ran through the rows of the attribute table, a second cursor compared the benchmark values with every value in all subsequent rows.

As the Python script ran through the rows, it compared whether or not different runs contained the same start and end benchmarks, or vice versa. Once the tool matched corresponding runs between surveys, the values of the elevation differences could be compared over time.

\subsubsection{Calculating Differences}

As the Python script located similar runs, a conditional statement compared the values of the differential leveling measurements. The cursors extracted this value from the table for the two matching rows, and then calculated the absolute value difference. The absolute value was used to rule out human error of data entry when the HGZ files were created.

The differential leveling value within the Runs feature class is stored as a float value with the field name "DifElev", and the units for this value are stored within the same tables as "DifElevUnit". The tool only asks for a float value for the significant difference value since the units may change between surveys. This requires the user to understand the units of the data when specifying a value, but all surveys within this pilot dataset used meters as the "DifElevUnit" value. Due to this, the tool did not differentiate any other measurement units.

\subsubsection{Detecting Changes in Height Differences}

Once the tool located similar runs and calculated the difference between the differential leveling values, it compared the resulting difference with the significant threshold specified by a user. The value specified by the user could be a float or integer value, to allow for higher precision. If the difference between the values was less or equal to the specified value, then the cursors continued running through the table. If the difference between the values was greater than the specified value, the tool wrote to the SigDifference table.

To visualize the runs with significant change over time, the table required certain field values such as a database key. The database key value extracted the ObjectID field 
from the table as the tool ran, and wrote this into the table as a RunID. This allowed the client to join the table with the Runs feature class to view the locations with significant elevation change.

The tool stored basic information about the matching runs to analyze the values of the differential leveling and when they occurred. The values stored within the table are as follows:

- Survey IDs of matching runs;

- Date of elevation measurement;

- Benchmark IDs of run;

- ObjectID from Runs table (to Join with Runs feature class);

- Differential leveling values from the two surveys;

- Difference between the differential leveling values;

The analysis tool allowed the client to locate and visualize areas of elevation change to understand two important factors: why there was an elevation change and what parts of the transportation infrastructure could be compromised.

\subsection{Conclusions}

This chapter covered the main processes used to create a GIS solution for the problem of the client. This project produced two different tools to assist the client in using and organizing their surveying datasets. The first tool automated the conversion of the client's pilot dataset into tables and feature classes within an ArcGIS 10.1 geodatabase. This was developed using Python and ESRI's ArcPy functionality. The second tool also used Python/ArcPy and completed temporal analysis on the dataset. The result was a table filled with runs that were measured over the same two benchmarks and had a significant elevation change over time. 



\section{Chapter 6 -Results: Use Case}

This project created a solution for the client to solve their spatial problem by creating geographic information from text files. The resulting product successfully converted these files into a geodatabase and created a spatial dataset which maintained all information from the original files.

\subsection{Graphical User Interface}

In order to simplify the conversion process for the client, who was not familiar with the use of Python scripts, an ArcGIS tool was created using a more familiar interface. As a regular GIS user, the client was now capable of accessing the geodatabase and using the conversion tool stored within it. The interface consisted of two user inputs: the Blue Book standard HGZ file and the location of the geodatabase. This graphical user interface (GUI) is shown in Figure 6.1.

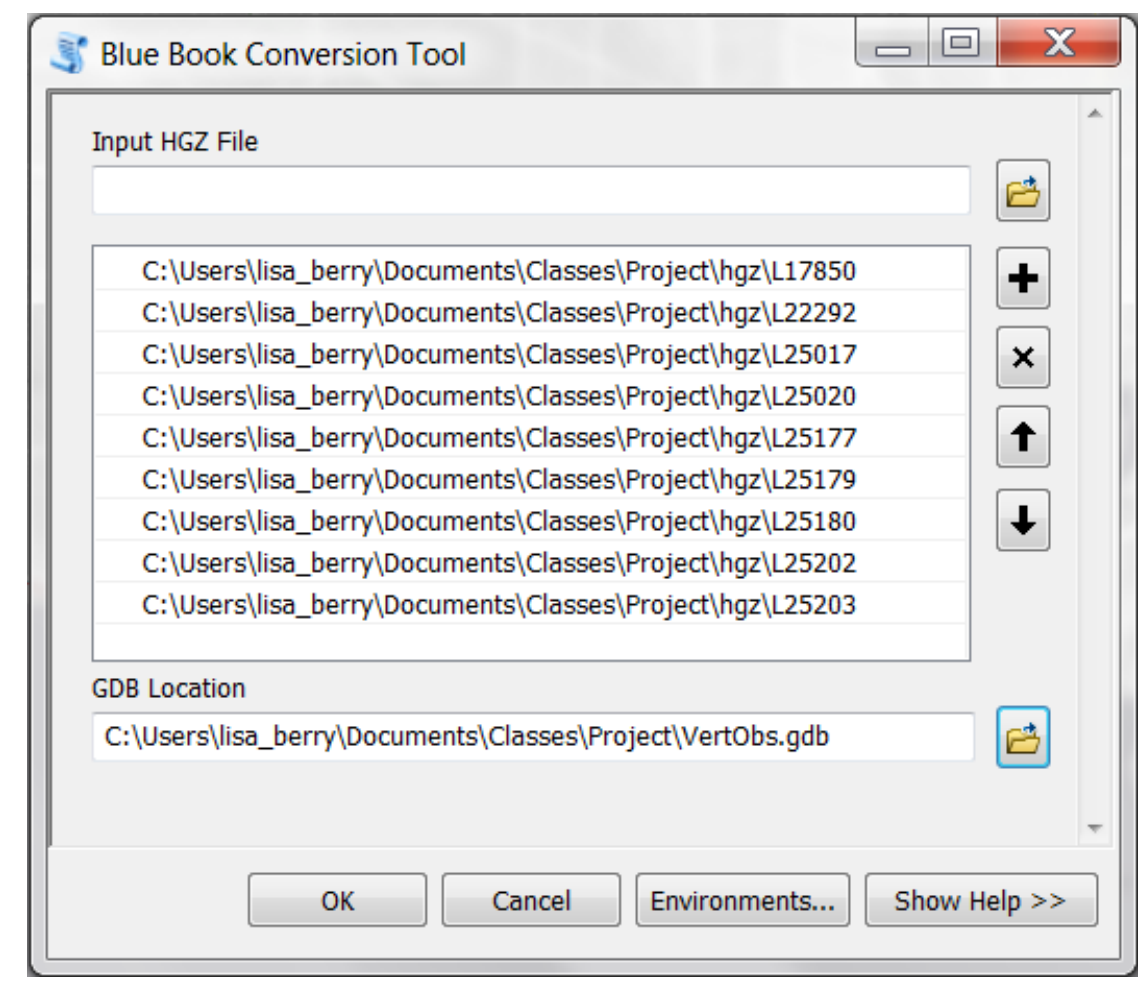

Figure 6.1: Graphical User Interface of the Conversion Toolset

Regular GIS users are familiar with this standard ArcGIS interface. The tool contains the same structure and process as the tools provided by the software itself. The use of this interface was also chosen because there is the ability to link the tool with a Python script. The use of batch processing allowed for multiple files to be added into the geodatabase at one time. 


\subsection{Updating the Geodatabase}

When the proper input files were used within the tool, the output resulted in the geodatabase tables being filled in less than 30 seconds for nine files. Figures 6.2 and 6.3 displays an example of two tables resulting from the conversion tool, and emphasizes important values created by the tool. The figure displays the spatial tables (Benchmarks and Runs) respectively.

\begin{tabular}{|c|c|c|c|c|c|c|}
\hline OBJECTID & SurveyID & PID* & SSN & Latitude_DD & Longitude_DD & Position \\
\hline 10692 & L25202 & EV9089 & 9967 & 34.10472 & -117.2939 & \\
\hline 10693 & L25202 & EV1065 & 9968 & 34.10472 & -117.2894 & \\
\hline 10694 & L25202 & EV1057 & 9969 & 34.10444 & -117.3033 & \\
\hline 10695 & L25202 & EV1054 & 5001 & 34.11195 & -117.3036 & \\
\hline 10696 & L25202 & EV1310 & 5002 & 34.125 & -117.3033 & \\
\hline 10697 & L25202 & EV9098 & 5003 & 34.13222 & -117.3086 & \\
\hline 10698 & L25202 & EV3392 & 5004 & 34.13611 & -117.3142 & \\
\hline 10699 & L25202 & EV1305 & 5005 & 34.13861 & -117.3175 & \\
\hline 10700 & L25202 & EV1301 & 5006 & 34.14833 & -117.3267 & \\
\hline 10701 & L25202 & EV1300 & 5007 & 34.15389 & -117.3314 & \\
\hline 10702 & L25202 & EV3393 & 5008 & 34.15944 & -117.3367 & \\
\hline 10703 & L25202 & EV1296 & 5009 & 34.17111 & -117.3464 & \\
\hline 10704 & L25202 & EV1292 & 5010 & 34.18111 & -117.3564 & \\
\hline 10705 & L25202 & EV1290 & 5011 & 34.18945 & -117.3639 & \\
\hline 10706 & L25202 & EV9099 & 5012 & 34.19083 & -117.3614 & \\
\hline 10707 & L25202 & EV9101 & 5014 & 34.1875 & -117.3531 & \\
\hline 10708 & L25202 & EV9100 & 5013 & 34.1875 & -117.3531 & \\
\hline 10709 & L25202 & EV9107 & 5015 & 34.18778 & -117.3475 & \\
\hline 10710 & L25202 & EV9102 & 5016 & 34.19278 & -117.3447 & \\
\hline 10711 & L25202 & EV9103 & 5017 & 34.19667 & -117.3411 & \\
\hline 10712 & L25202 & EV9104 & 5018 & 34.19944 & -117.3383 & \\
\hline 10713 & L25202 & EV9105 & 5019 & 34.20195 & -117.3331 & \\
\hline 10714 & L25202 & EV9106 & 5020 & 34.1825 & -117.3725 & \\
\hline 10715 & L25202 & EV9108 & 5021 & 34.17916 & -117.3822 & \\
\hline 10716 & L25202 & EV9109 & 5022 & 34.17639 & -117.3883 & \\
\hline & Datab & ase $\mathrm{Ke}$ & & \multicolumn{2}{|c|}{ Spatial Data } & Null Data \\
\hline
\end{tabular}

Figure 6.2: Benchmarks Table Attributes After Running Conversion Tool 


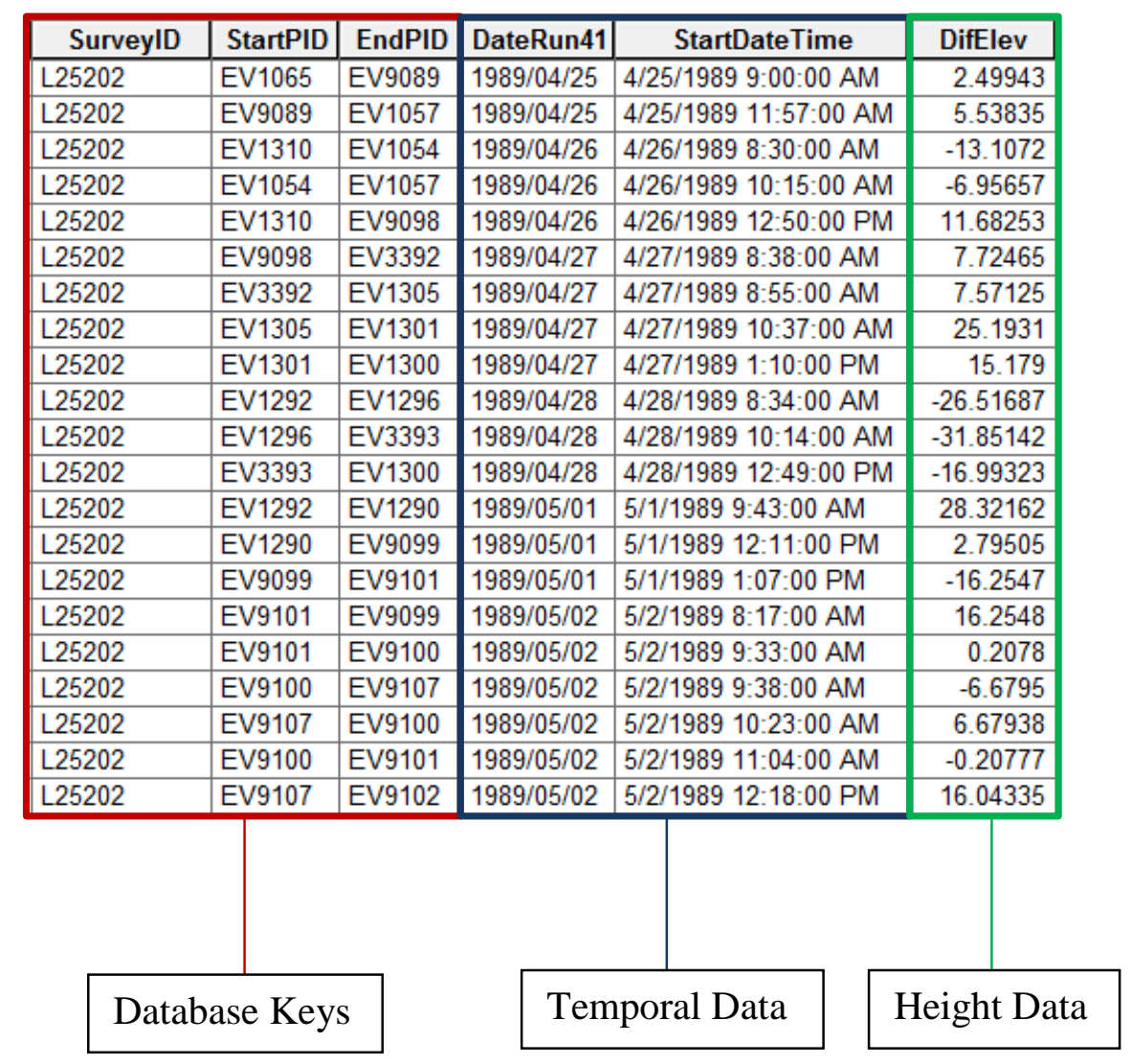

Figure 6.3: Runs Table Attributes After Running Conversion Tool

As seen in the figure, the conversion tool created different data types to match various fields within ArcMap. Since the original dataset was all in text format, some fields remained text and if they were null values, they would show up blank. Otherwise, integer, date, and spatial fields were created to make the dataset display within a GIS setting. The majority of the dataset was placed into these two feature classes and therefore most of the analyses would be performed using these tables.

Non-spatial tables were also a part of the geodatabase, and were filled using the same conversion tool. These tables, if necessary for the client, can be linked to other tables using the highlighted database key. An example of this is shown in Figure 6.4. 


\begin{tabular}{|r|l|l|l|l|l|l|}
\hline OBJECTID & SurveyID & Vertobs & Agency & \multicolumn{1}{|c|}{ Agency } & DateCreated & Version \\
\hline 91 & L25202 & VERTOBS & MWDSC & METRO WATER DISTR SO. CALIFORNIA & $2013 / 03 / 26$ & 4.042 \\
\hline 92 & L25177 & VERTOBS & NGS & NATIONAL GEODETIC SURVEY & $2013 / 03 / 26$ & 4.043 \\
\hline 93 & L17850 & VERTOBS & NGS & NATIONAL GEODETIC SURVEY & $2013 / 03 / 26$ & 4.027 \\
\hline 94 & L22292 & VERTOBS & NGS & NATIONAL GEODETIC SURVEY & $2013 / 03 / 26$ & 4.032 \\
\hline 95 & L25017 & VERTOBS & NGS & NATIONAL GEODETIC SURVEY & $2013 / 03 / 26$ & 4.040 \\
\hline 96 & L25020 & VERTOBS & NGS & NATIONAL GEODETIC SURVEY & $2013 / 03 / 26$ & 4.043 \\
\hline 97 & L25177 & VERTOBS & NGS & NATIONAL GEODETIC SURVEY & $2013 / 03 / 26$ & 4.043 \\
\hline 98 & L25179 & VERTOBS & CA1980 & CITY OF LOS ANGELES CALIFORNIA & $2013 / 03 / 26$ & 4.043 \\
\hline 99 & L25180 & VERTOBS & NGS & NATIONAL GEODETIC SURVEY & $2013 / 03 / 26$ & 4.043 \\
\hline 100 & L25202 & VERTOBS & MWDSC & METRO WATER DISTR SO. CALIFORNIA & $2013 / 03 / 26$ & 4.042 \\
\hline 101 & L25203 & VERTOBS & NGS & NATIONAL GEODETIC SURVEY & $2013 / 03 / 26$ & 4.043 \\
\hline
\end{tabular}

Database Key

Figure 6.4: Non-Spatial Table within Geodatabase and Key Value

\subsection{Point and Line Features}

After running the tool with the client's pilot dataset, all tables and feature classes were updated with the information from the input text files. The point and line feature classes could be displayed within ArcMap. While all surveys were inserted into the same tables and feature classes, using proper the symbology allowed individual surveys to be represented separately. Figure 6.5 shows the representation of the different surveys by Benchmarks (points) and Runs (lines). 


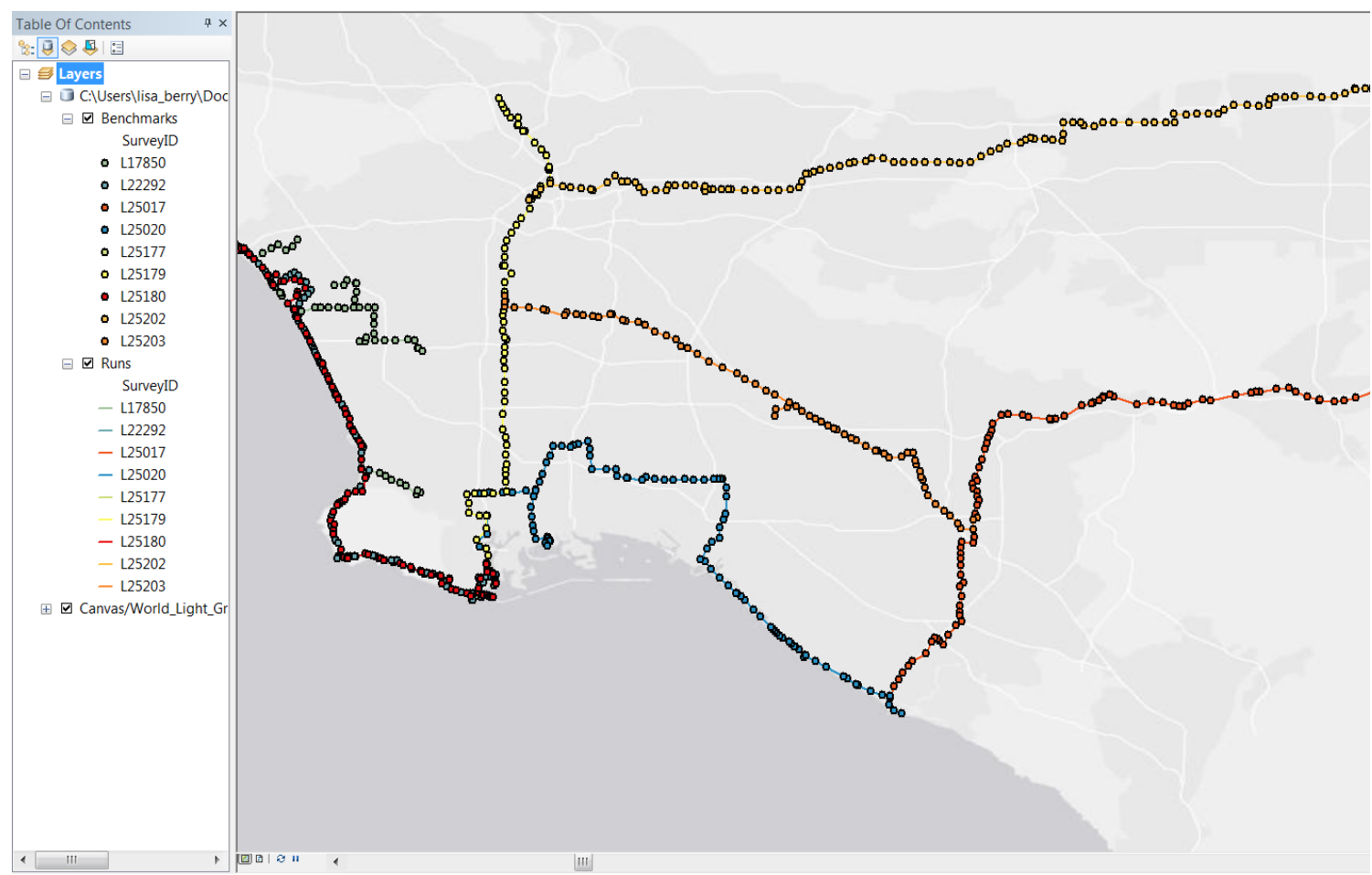

Figure 6.5: Point and Line Features Created By Tool

Point and line features from the same survey could be selected using a Select By Attributes selection. Figure 6.6 shows the Select By Attribute selection of two surveys and Figure 6.7 shows the two surveys being selected from the benchmarks layer as the highlighted blue values. 


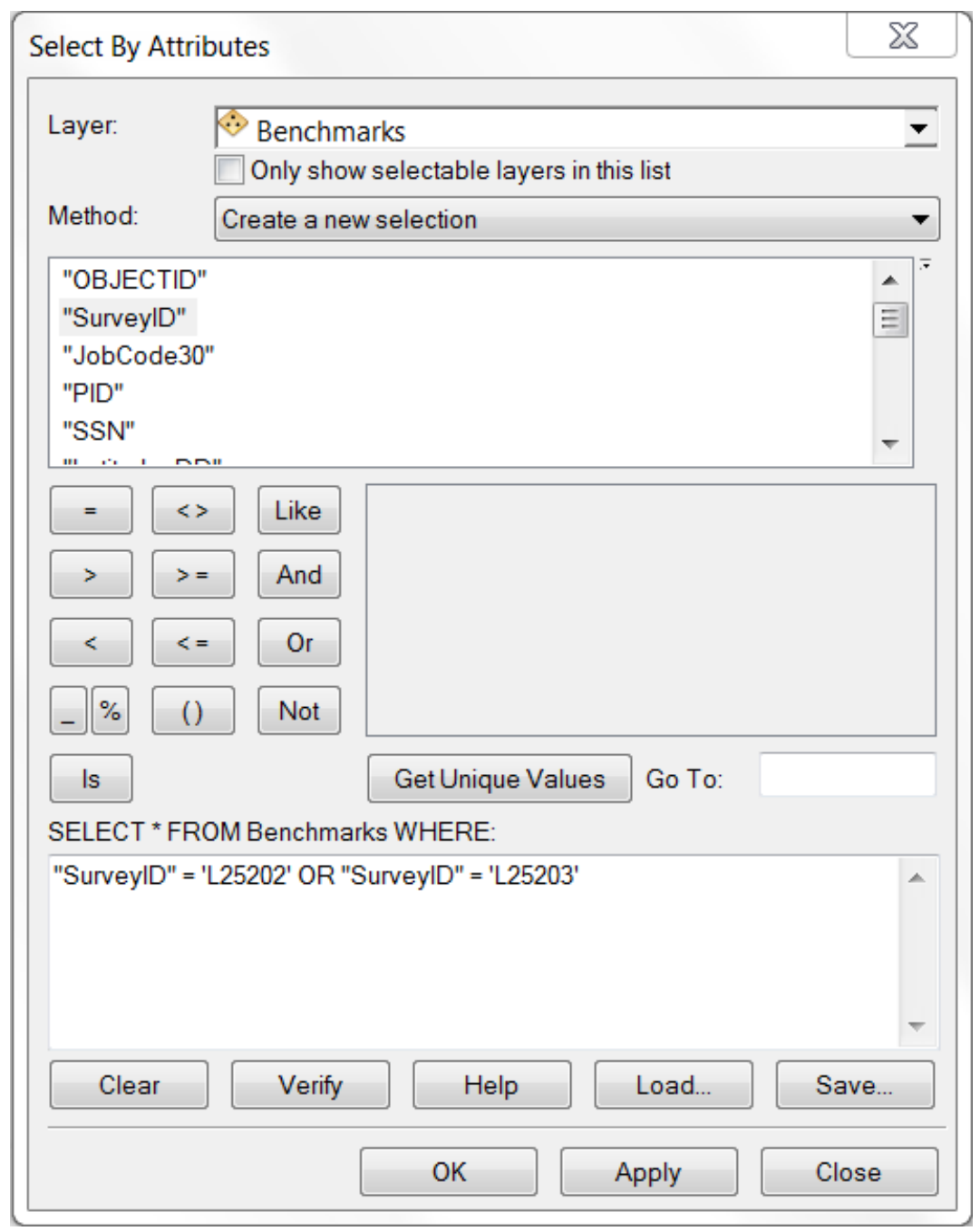

Figure 6.6: Select By Attributes Use Case 


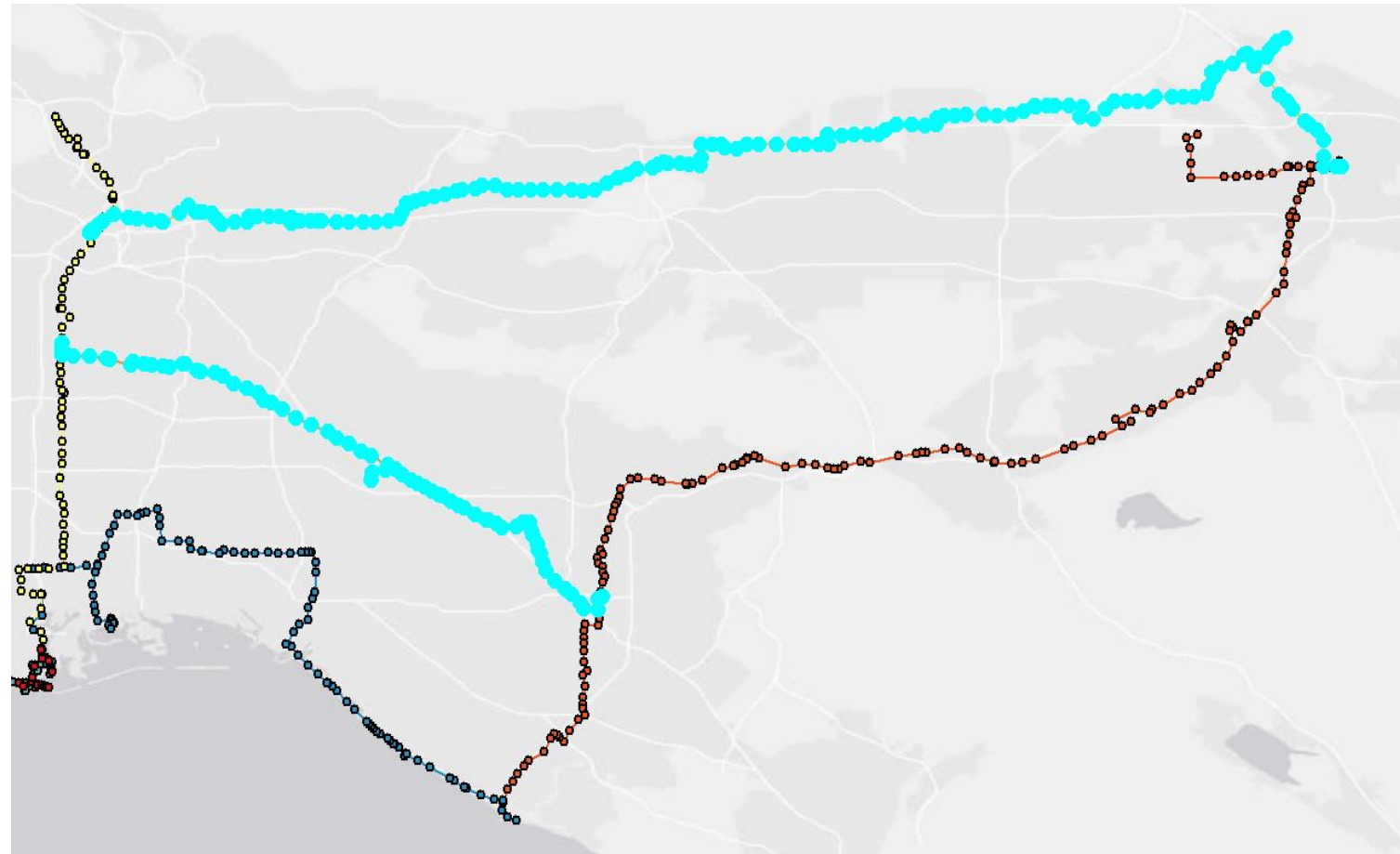

Figure 6.7: Select By Attribute Use Case Result

\subsection{Differential Height Differences Tool}

As explained in chapter 5, the temporal analysis tool performed a comparison analysis on the resulting Runs feature class output from the conversion tool. This analysis compared the differential leveling height differences over different dates. The result was a table within the geodatabase which could be joined with the Runs feature class and represented as runs (line segments). Figure 6.8 shows the interface of the analysis tool, which is stored within the geodatabase.

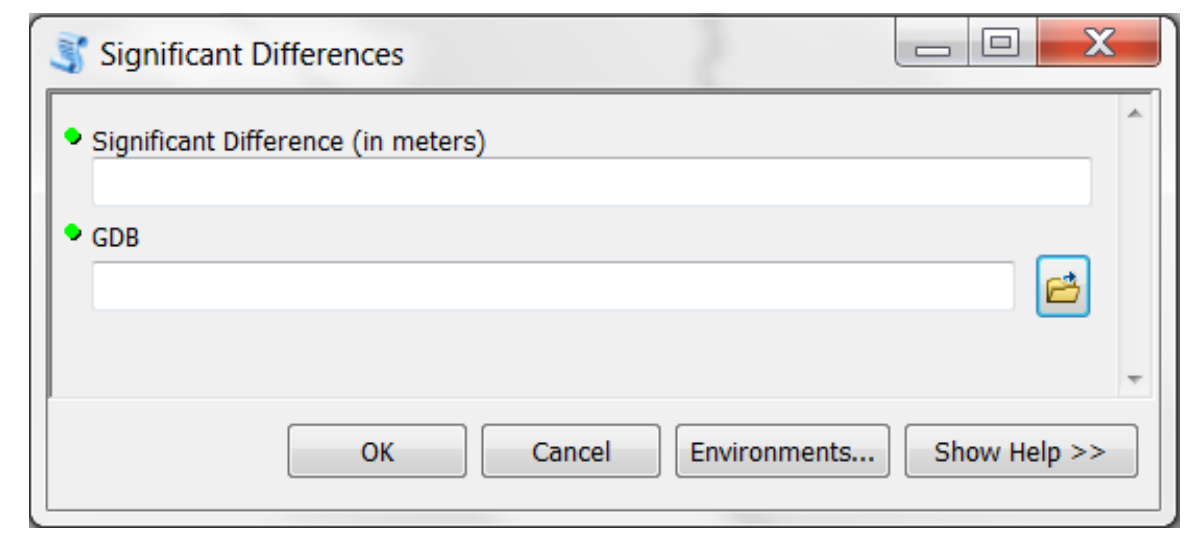

Figure 6.8: Significant Differences Temporal Analysis Tool

The user is asked to specify a significant difference threshold in elevation, and the resulting table is filled with all run values which have a difference in elevation larger than 
the specified value. As seen in Figure 6.8, this value is the "Significant Difference" value. The tool required the user to specify the significant difference value, and the location of the geodatabase. The result of this tool fills the SigDifference table with the values explained in Chapter 5. Figure 6.9 shows an example of the result of the tool using .03 as the significant difference value.

\begin{tabular}{|c|c|c|c|c|c|c|c|c|c|}
\hline SigValue & PID_1 & PID_2 & RunID_1 & RunID_2 & Survey_1 & Survey_2 & Date_1 & Date_2 & Difference \\
\hline 0.03 & DZ0814 & DZ0815 & 17434 & 17434 & L25177 & L22292 & $1989 / 05 / 03$ & $1970 / 10 / 06$ & 0.03277 \\
\hline 0.03 & DZ0814 & DZ0815 & 17434 & 17434 & L25177 & L22292 & $1989 / 05 / 03$ & $1970 / 10 / 06$ & 0.03307 \\
\hline 0.03 & DZ0972 & DZ0971 & 17566 & 17566 & L25177 & L25177 & $1989 / 05 / 19$ & $1989 / 07 / 20$ & 0.36712 \\
\hline 0.03 & DZ0972 & DZ0971 & 17566 & 17566 & L25177 & L25177 & $1989 / 05 / 19$ & $1989 / 07 / 20$ & 0.36677 \\
\hline 0.03 & DZ0972 & DZ0971 & 17566 & 17566 & L25177 & L22292 & $1989 / 05 / 19$ & $1970 / 11 / 03$ & 0.36297 \\
\hline 0.03 & DZ0972 & DZ0971 & 17566 & 17566 & L25177 & L22292 & $1989 / 05 / 19$ & $1970 / 11 / 03$ & 0.36307 \\
\hline 0.03 & DZ0972 & DZ0971 & 17566 & 17566 & L25177 & L25177 & $1989 / 05 / 19$ & $1989 / 07 / 20$ & 0.36712 \\
\hline 0.03 & DZ0972 & DZ0971 & 17566 & 17566 & L25177 & L25177 & $1989 / 05 / 19$ & $1989 / 07 / 20$ & 0.36677 \\
\hline 0.03 & DZ0072 & DZ0071 & 17657 & 17657 & L25177 & L22292 & $1989 / 06 / 05$ & $1971 / 01 / 27$ & 0.05935 \\
\hline 0.03 & DZ0072 & DZ0071 & 17657 & 17657 & L25177 & L22292 & $1989 / 06 / 05$ & $1971 / 01 / 27$ & 0.05905 \\
\hline 0.03 & EW3845 & EW3850 & 17714 & 17714 & L25177 & L22292 & $1989 / 06 / 09$ & $1971 / 02 / 11$ & 0.06903 \\
\hline 0.03 & EW3845 & EW3850 & 17714 & 17714 & L25177 & L22292 & $1989 / 06 / 09$ & $1971 / 02 / 11$ & 0.06958 \\
\hline 0.03 & EW3787 & EW3791 & 17738 & 17738 & L25177 & L22292 & $1989 / 06 / 13$ & $1971 / 02 / 16$ & 0.0365 \\
\hline 0.03 & EW3787 & EW3791 & 17738 & 17738 & L25177 & L22292 & $1989 / 06 / 13$ & $1971 / 02 / 16$ & 0.0372 \\
\hline 0.03 & DZ0971 & DZ0972 & 17749 & 17749 & L25177 & L25177 & $1989 / 07 / 20$ & $1989 / 05 / 19$ & 0.36712 \\
\hline 0.03 & DZ0972 & DZ0971 & 17750 & 17750 & L25177 & L25177 & $1989 / 07 / 20$ & $1989 / 05 / 19$ & 0.36677 \\
\hline 0.03 & DY1229 & DY1230 & 17861 & 17861 & L17850 & L25180 & $1960 / 04 / 20$ & $1989 / 07 / 10$ & 0.439099 \\
\hline 0.03 & DY1230 & DY1229 & 17865 & 17865 & L17850 & L25180 & $1960 / 04 / 20$ & $1989 / 07 / 10$ & 0.4361 \\
\hline 0.03 & DY1240 & DY1239 & 17867 & 17867 & L17850 & L22292 & $1960 / 04 / 21$ & $1971 / 03 / 22$ & 0.1819 \\
\hline 0.03 & DY1240 & DY1239 & 17867 & 17867 & L17850 & L22292 & $1960 / 04 / 21$ & $1971 / 03 / 22$ & 0.18155 \\
\hline 0.03 & DY1235 & DY1233 & 17870 & 17870 & L17850 & L25180 & $1960 / 04 / 21$ & $1989 / 07 / 10$ & 0.0406 \\
\hline 0.03 & DY1233 & DY1235 & 17875 & 17875 & L17850 & L25180 & $1960 / 04 / 21$ & $1989 / 07 / 10$ & 0.041899 \\
\hline 0.03 & DY1239 & DY1240 & 17878 & 17878 & L17850 & L22292 & $1960 / 04 / 21$ & $1971 / 03 / 22$ & 0.1835 \\
\hline 0.03 & DY1239 & DY1240 & 17878 & 17878 & L17850 & L22292 & $1960 / 04 / 21$ & $1971 / 03 / 22$ & 0.18315 \\
\hline 0.03 & DY1187 & DY1189 & 17925 & 17925 & L17850 & L25180 & $1960 / 04 / 26$ & $1989 / 07 / 07$ & 0.0724 \\
\hline
\end{tabular}

Figure 6.9: SigDifference Table Filled with Analysis Tool

The tool itself fills this table, but the user must complete additional steps to analyze and visualize the table further. Since the table contains a RunID for each run which matches the ObjectID in the Runs table, it can use a Join to connect the table with the Runs feature class. Once the table is joined, a query can be used to select runs with a certain difference value or range. The selected values can then be exported to a new map layer and visualized. Figure 6.10 shows a zoomed-in region of this resulting layer from the pilot dataset with a difference value of .03 meters or greater. This visualization shows the differentiation between smaller and larger differences to allow Caltrans to focus on major height changes. 


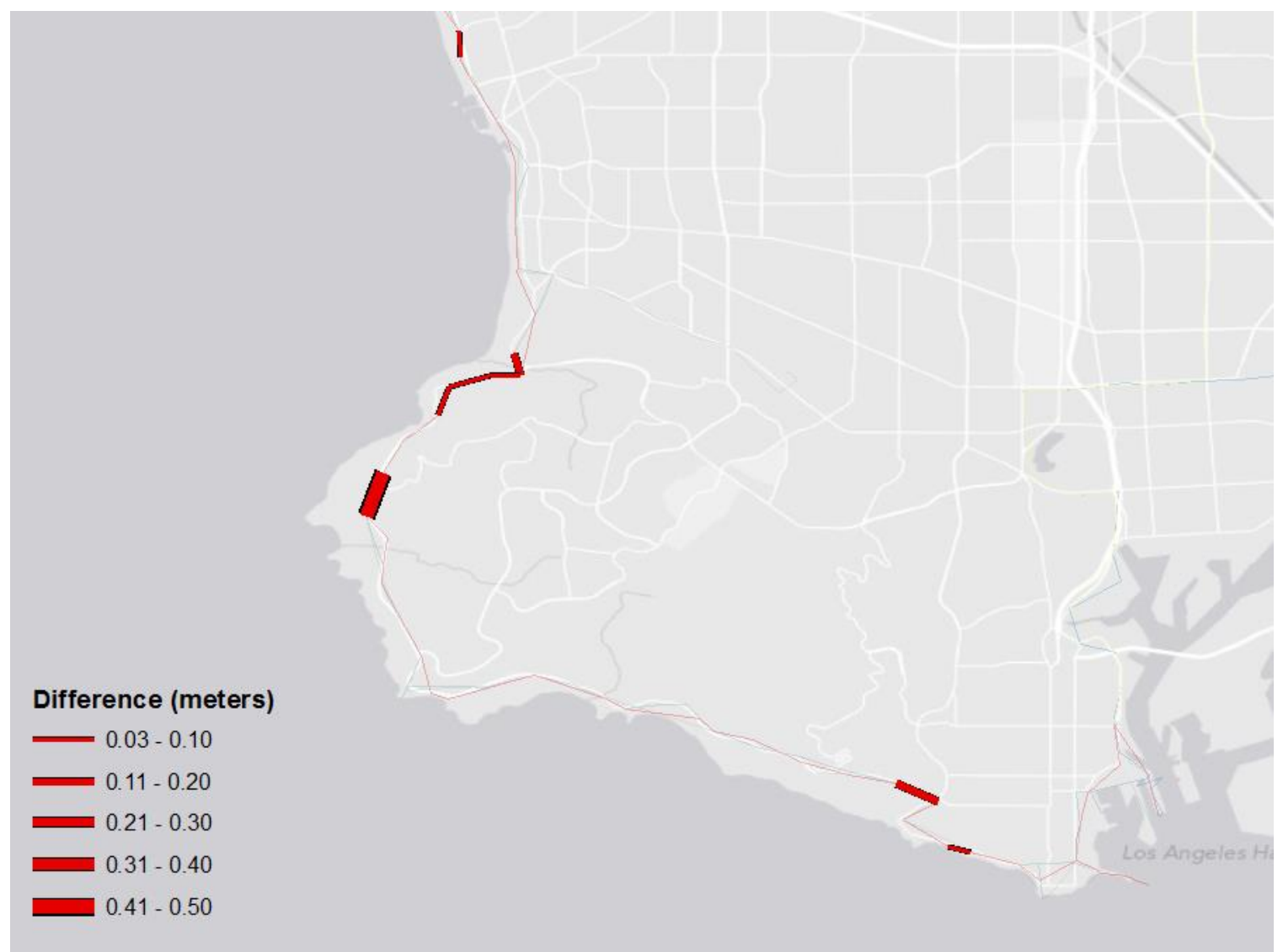

Figure 6.10: Highlighted Runs with Significant Differences over Time

\subsection{Uses of the Result}

The conversion tool efficiently stores the survey observation data into a geodatabase and ultimately allows for many different forms of analyses. The client or any other user is now capable of performing analyses using the raw observations, and can compute various different values using the geodatabase.

The client also gained the ability to overlay geological features to determine if areas of height change correspond with geomorphological phenomena. While this project did not perform any of this analysis, Figures $6.11,6.12$, and 6.13 display the ability to overlay these geological layers. 


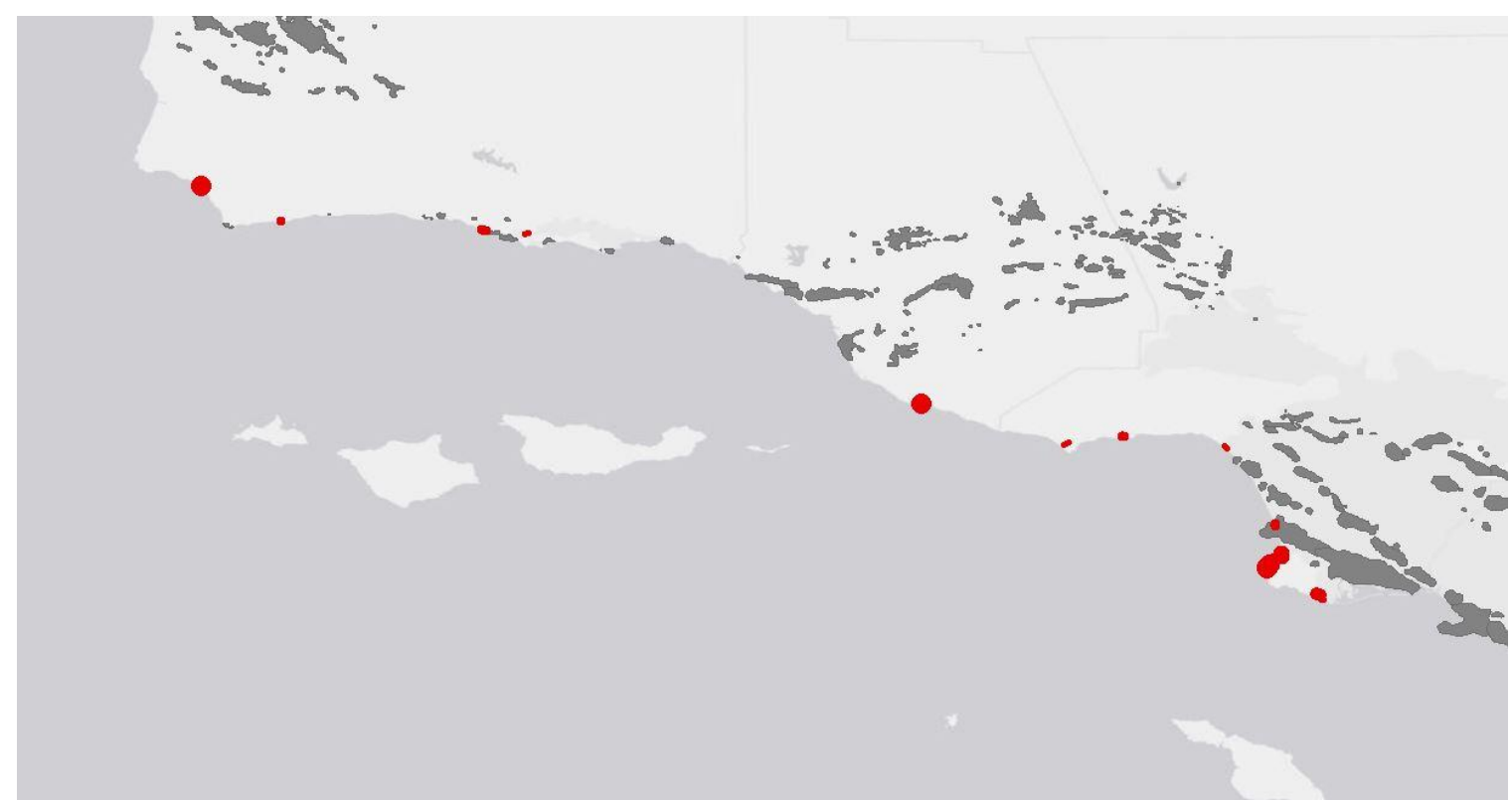

Figure 6.11: Oil and Gas Basin Overlay with Height Change Results

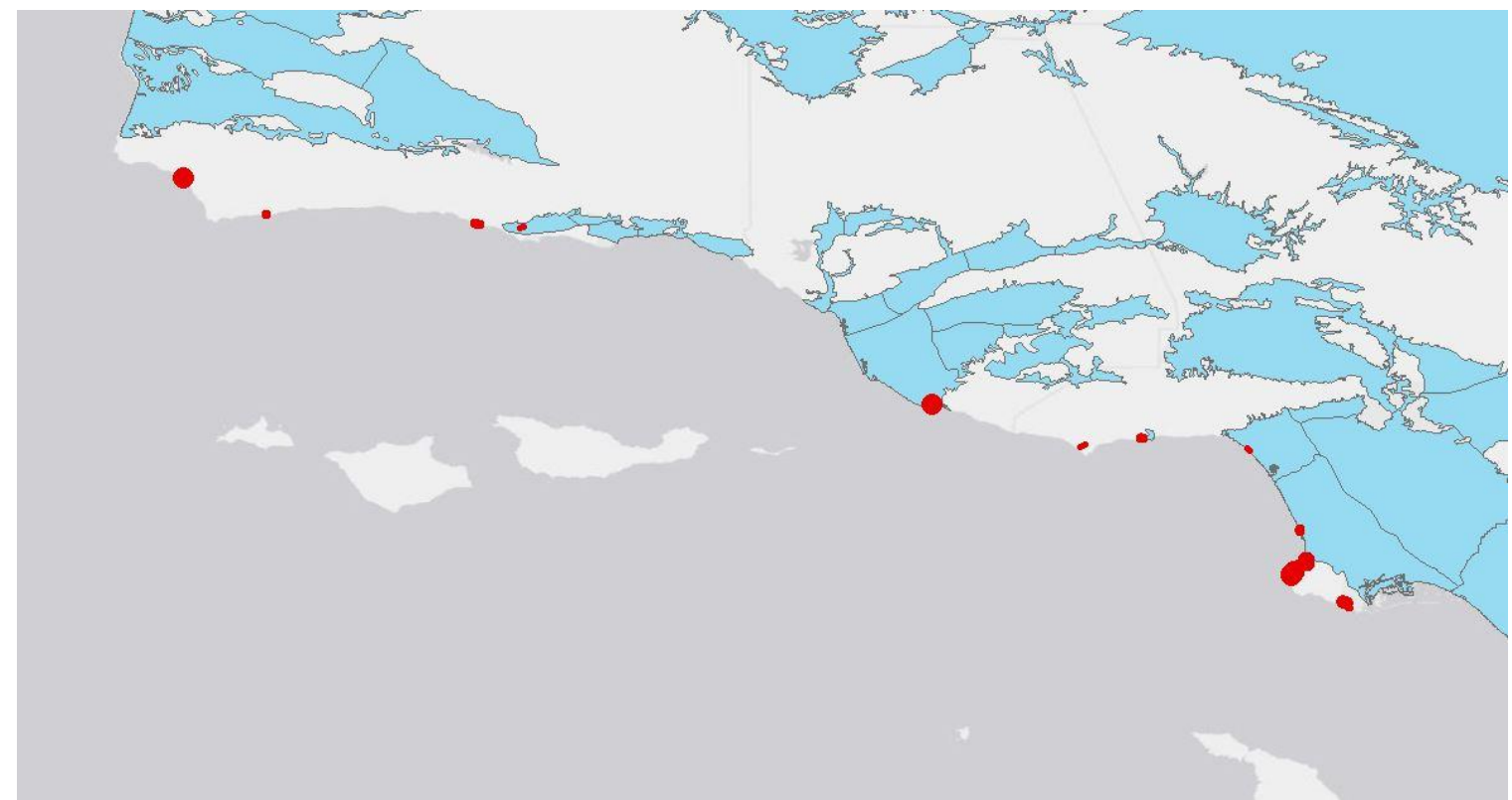

Figure 6.12: Water Basin Overlay with Height Change Results 


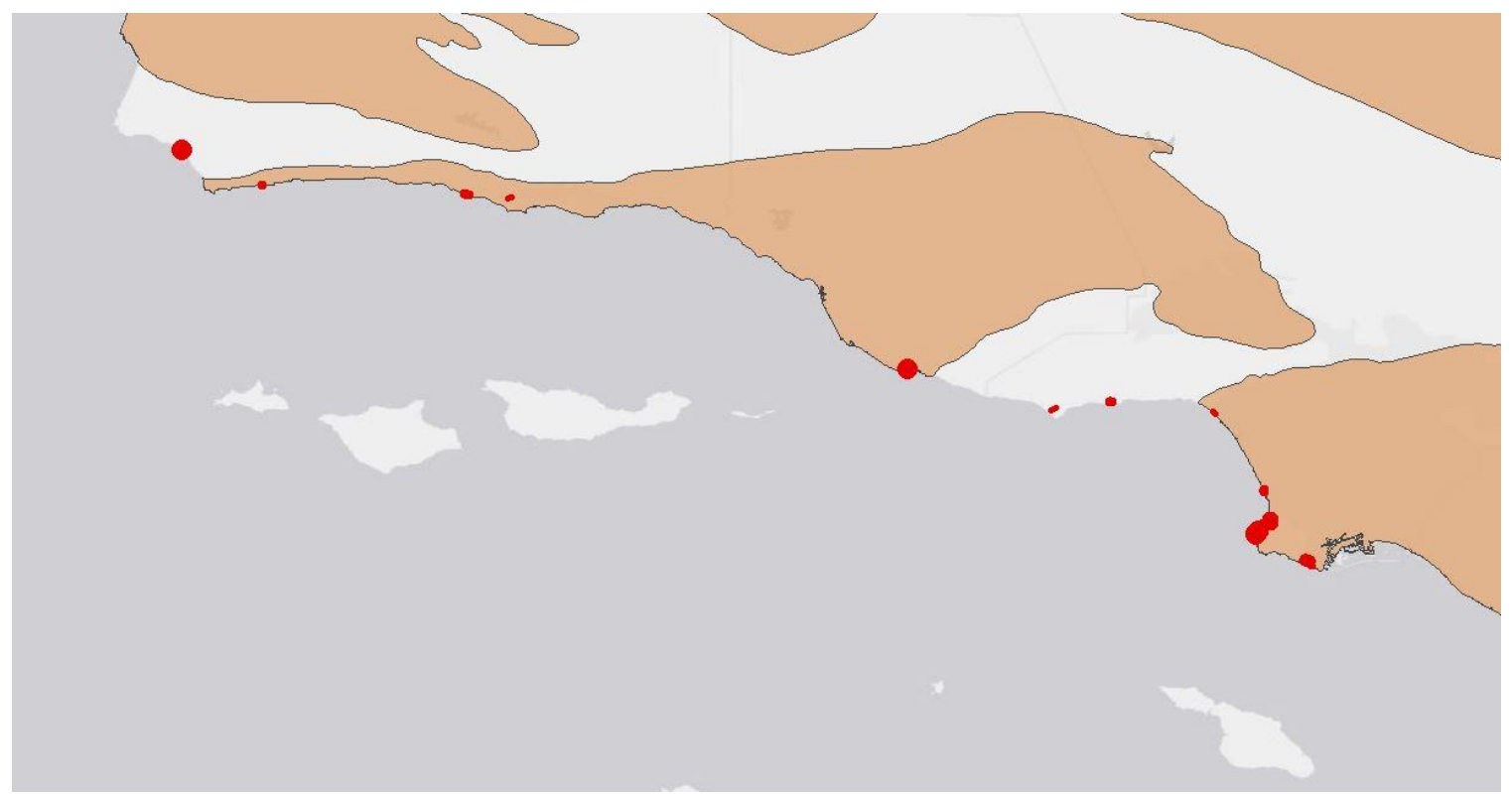

Figure 6.13: Sediment Basin Overlay with Height Change Results

\subsection{Summary}

The tools created in this project allowed the client to utilize their datasets in multiple different ways. Centralized storage within a geodatabase allows for easier access and analysis of the raw survey information. The use of a GIS provides the ability to visualize, overlay, and perform spatial calculations with the datasets. Overall, this project not only allows the client to organize their datasets, but the conversion tool allows their analyses to expand as they continue to add additional datasets. 



\section{Chapter 7 -Conclusions and Future Work}

\subsection{Conclusions}

Recognizing elevation changes in height over time allows Caltrans to direct funding and attention to areas of need along the transportation infrastructure in southern California. The inability to efficiently use NGS formatted HGZ files containing differential leveling surveys made analysis of these elevation changes tedious and expensive. This project automated the conversion of the HGZ text files into a spatial database organized format to allow for temporal analyses.

Python and ArcPy methods were used to read the files provided by the client and create a geodatabase structure to store the information. By converting and processing every attribute within the text files, an operational geodatabase schema was updated with Blue Book standardized input files. The automation of the process creates the ability to analyze and overlay surveys with geomorphological features.

Overall, this project created a spatial solution for the client to utilize their NGS Blue Book datasets. By meeting the functional and non-functional requirements of the client, they were given the ability to use additional NGS survey files to complete spatial and temporal analyses. The ability to perform this analysis enables Caltrans to closely analyze the southern California region over time. This project allows for the identification of areas with large height change. Recognizing these areas permits Caltrans to overlay their datasets with geomorphological layers to identify cause and effect of the temporal changes. All of these newfound abilities give Caltrans a better sense of where to focus their attentions on the transportation infrastructure.

This project involved a large amount of Python scripting, which required a thorough understanding of ArcPy and debugging coding methods. As the script was created, the process of learning these methods and using them added another value created from this project. The ability to learn while creating a GIS solution was a valuable lesson and can used in the field of GIS with most projects. Overall, this project created value for not only the client, but also for the creator.

\subsection{Future Work}

The file geodatabase used within this project allows the client to store their VERT OBS datasets within a spatial database and complete analysis on the data temporally. The client would like to further use these datasets as a network and determine if various surveys complete a looped network. This would require network or linear referencing to organize the elevation datasets for benchmark heights. A project involving much larger datasets would allow this analysis to be completed and could be considered future work for the client or any user of the Blue Book formatted datasets.

Another possibility for future work on this project would be to create a web application which provides the conversion tool within a map online. This web map could display any new datasets in the map as they are input. A tool such as this could allow multiple files to be used as inputs and instantly display the output within a web 
application. Additionally, the web application could use geoprocessing tools to perform the temporal and network analysis for the input survey datasets within a web browser.

The geodatabase for this project could also be expanded to contain another tool which performs all of the necessary steps to complete analysis. This would reduce the amount of work a user would have to perform to complete analysis on the datasets after using the conversion tool alone.

Overall, the project could be perfected and tweaked until the quality of the tool handled every type of possible input Blue Book file. This could be completed with a more thorough dataset, and would require additional Python coding and geodatabase creation. 


\section{Works Cited}

Butler, A. J. (2008). Designing Geodatabases for Transportation. Redlands: ESRI Press. California Department of Transportation. (2006, September). Chapter 8: Differential Leveling Survey Specifications . Retrieved Frebruary 8, 2013, from California Department of Transportation Surveys Manual: http://www.dot.ca.gov/hq/row/landsurveys/SurveysManual/08_Surveys.pdf

Estopinal, S. V. (2009). A Guide to Understanding Land Surveys. New Jersey: John Wiley \& Sons, Inc.

Leach, T. C. (2007, August). A Geodatabase for the Washington Council of County Surveyors Data Warehouse. Redlands: University of Redlands.

National Geodetic Survey. (2001). Reduce Record Format. National Oceanic and Atmospheric Administration .

National Oceanic and Atmospheric Administration. (1992, December). VFPROC: Vertical Control Field Data Processing System. Retrieved November 13, 2013, from National Geodetic Survey: http://www.ngs.noaa.gov/PUBS_LIB/VFPROC.pdf

National Oceanic and Atmospheric Administration. (2004, November 24). Input Formats and Specifications of the National Geodetic Survey Data Base. Retrieved October 15, 2013, from National Oceanic and Atmospheric Administration: http://www.ngs.noaa.gov/FGCS/BlueBook/pdf/Chapter6.pdf

Satalich, J. (2010). Analysis and Readjustment of Differential Leveling Obersvations from SR 92-121. Los Angeles: California Department of Transportation.

Snay, R., Cline, M., Dillinger, W., Foote, R., Hilla, S., Kass, W., et al. (2007). Using Global Positioning System-dreived Crustal Velocities to Estimate Rates of Absolute Sea Level Change from North American Tide Gauge Records. Journal of Geophysical Research, 112(B04409).

Stein, R. S., Whalen, C. T., Holdahl, S. R., Strange, W. E., \& Thatcher, W. (1986). Saugus-Palmdale, California, Field Test for Refraction Error in Historical Leveling Surveys. Journal of Geophysical Research, 91(B9), 9031-9044.

Torres-Ruiz, G. L. (2009, August). Web-based GIS Application for Dissemination of Geodetic Data of Military Airfields. Redlands: University of Redlands.

Transportation, T. D. (2011, April). Differential Leveling. Retrieved December 2013, from TxDOT Survey Manual:

http://onlinemanuals.txdot.gov/txdotmanuals/ess/differential_leveling.htm 



\section{Appendix A. VertobsConversion.py}

This script is the main conversion script used to convert the HGZ files into the geodatabase. It is commented to describe most functionalities, but many of the processes are described within Chapter 5. This tool links to the Blue Book Conversion tool and writes to the Blue Book geodatabase. 


\section{Appendix B. SignificantDifference.py}

This code is the Python script connected to the Significant Differences tool within the geodatabase. This analysis tool runs multiple ArcPy cursors in order to read and write to tables within the geodatabase for this project. This script links to the "Significant Differences" Tool and writes to the SigDifference table. 


\section{Appendix C. Reduce (Reduc6) Format Specifications}

As described in Chapter 2, this is the Reduce key used to determine the line indexing for the conversion tool. This follows the Blue Book Vert Obs specifications.

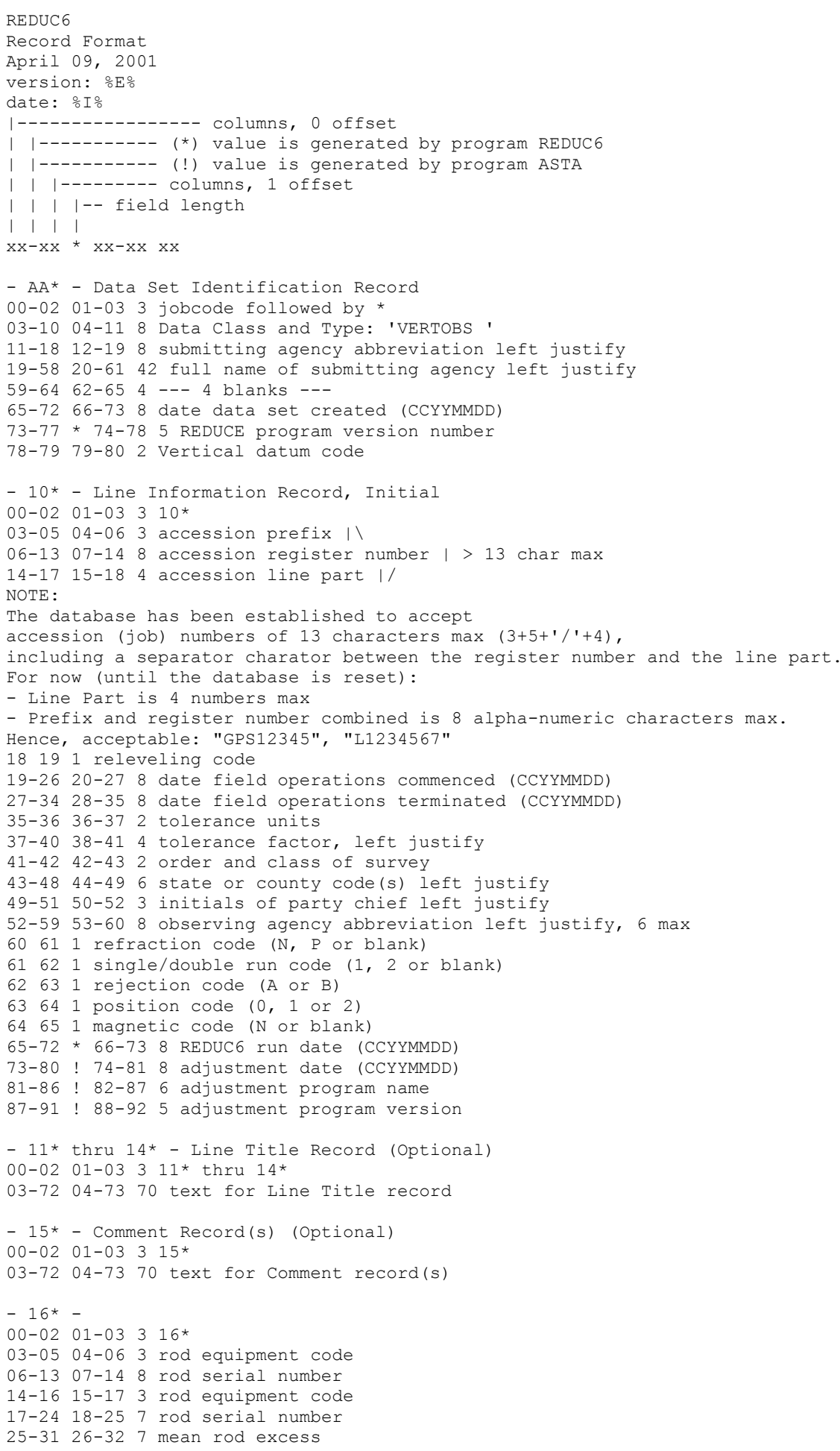




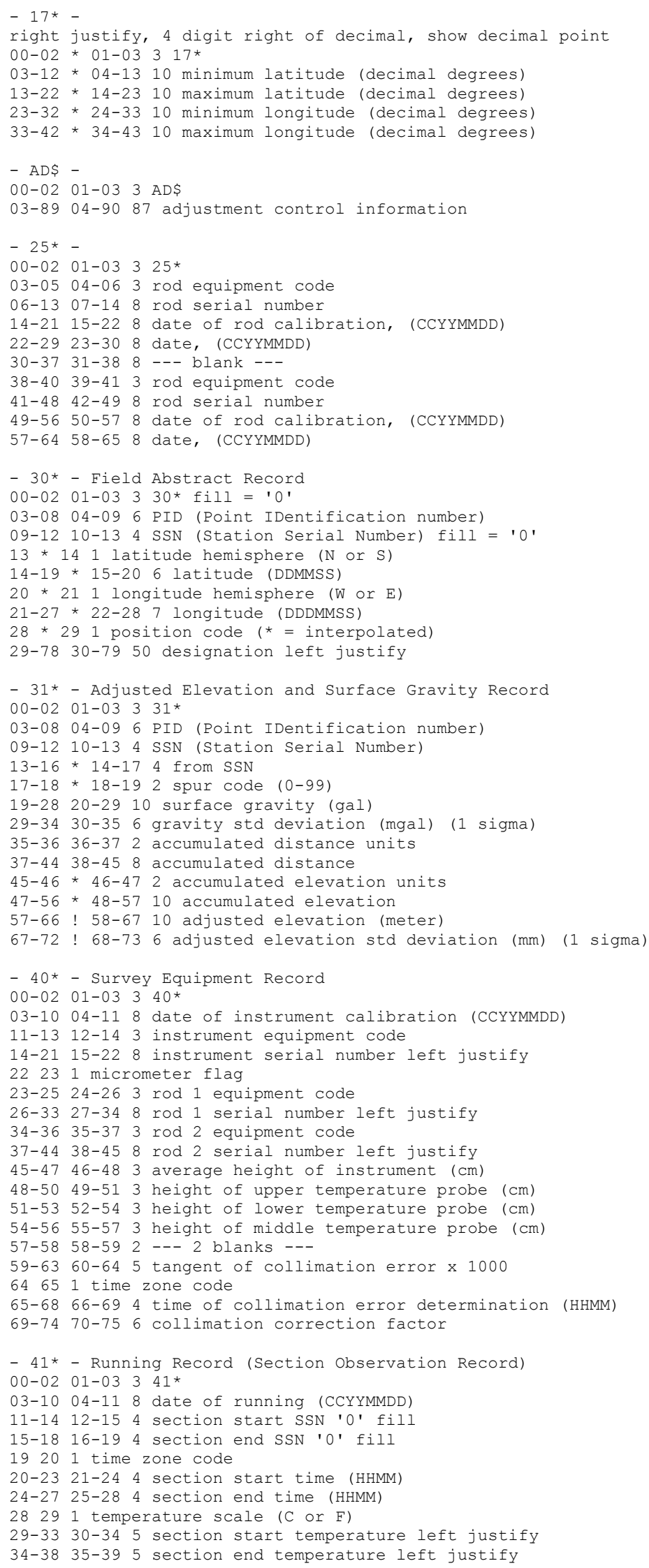


39401 wind code

40411 sun code

41-43* 42-44 3 number of setups in this section

$44 * 451$ setup code ( $\mathrm{E}=$ estimated)

45461 stadia intercept code

46-50 47-51 5 stadia interval sum, backsight

51-55 52-56 5 stadia interval sum, foresight

$56-57 * 57-582$ running length units

$58-62$ *59-63 5 running length left justify

63-64 64-65 2 elevation difference units

65-74 66-75 10 elevation difference left justify

75-77 76-78 3 initials of survey crew chief left justify

$78-83$ * 79-84 6 normal orthometric correction (mm)

84-88 85-89 5 observation id

- 42* - River/Valley Crossing (Observation) Record

$00-02 \quad 01-03 \quad 3 \quad 42 *$

03-10 04-11 8 date of river/valley crossing (CCYYMMDD)

11-14 12-15 4 section start SSN

15-18 16-19 4 section end SSN

19201 time zone code

20-23 21-24 4 section start time (HHMM)

24-27 25-28 4 section end time (HHMM)

28-55 29-56 28 --- blank --

56-57 57-58 2 river/valley crossing length units

58-62 59-63 5 river/valley crossing length

63-64 64-65 2 elevation difference units

65-74 66-75 10 elevation difference

$75-77$ 76-78 3 --- blank ---

$78-83$ * 79-84 6 normal orthometric correction (mm)

84-88 85-89 5 observation id

- 43* - Observation and Correction Record

$00-0201-03343 *$

03-10 04-11 8 date of running (CCYYMMDD)

11-14 12-15 4 section start SSN

15-18 16-19 4 section end SSN

19-22 20-23 4 section start time (HHMM)

23-27 24-28 5 mean temperature for upper probe

28-32 29-33 5 mean temperature for lower probe

33-37 34-38 5 mean temperature for middle probe

38391 temperature scale

39401 temperature code (predicted, observed)

40 * 411 refraction/astronomic/magnetic (RAM) code (0-7)

$41 * 421$ rejection flag

42-48* 43-497 refraction correction (mm)

49 * 501 refraction correction method (Precomputed/Reduc6)

50-56*51-57 7 rod correction (mm)

57 * 581 rod correction method (Precomputed/Reduc6)

$58-63 * 59-646$ level correction (mm)

64-69*65-70 6 temperature correction (mm)

$70-75 * 71-766$ astronomic correction $(\mathrm{mm})$

$76-81 * 77-826$ magnetic correction (mm)

82-90* 83-91 9 partial refraction correction (units?)

- 44* - Adjusted Elevation Statistics Record

$00-0201-03 \quad 344 *$

03-10 04-11 8 date of running (CCYYMMDD)

11-14 12-15 4 section start SSN

15-18 16-19 4 section end SSN

19-22 20-23 4 section start time (HHMM)

$23 * 241$ rejection code

$24-33 * 25-3410$ residual $(\mathrm{mm})$

34-43* 35-44 10 std deviation of adjusted obs (mm) (unscaled)

- 65* - Line Information Record, Completion

$00-02 * 01-03365 *$

03-08* 04-09 6 std deviation for $1 \mathrm{~km}$ single run section (mm) (1 sigma)

09-14* 10-15 6 std deviation for $1 \mathrm{~km}$ double run section (mm) (1 sigma)

$15-22 * 16-238$ main line length $(\mathrm{km})$

$23-30 * 24-318$ length of $\operatorname{spurs}(\mathrm{km})$

31-38*32-39 8 length of accepted leveling $(\mathrm{km})$

$39-43 * 40-445$ number of sections

$44-48 * 45-495$ number of runnings

$49-53 * 50-545$ number of river/valley crossings

$54-58 * 55-595$ number of bench marks

$59-63 * 60-645$ number of temporary bench marks

64-68*65-695 percentage of rerun sections, including rejected sections

69-74! 70-75 5 degrees of freedom in adjustment

75-80! 76-81 6 number of unknowns in adjustment

81-89! 82-90 9 aposteriori variance of unit weight from adjustment 
- AA* - Data Set Termination Record 00-02 01-03 3 jobcode followed by * 